\title{
Differential role of spinal and supraspinal processing of proprioceptive information in mouse locomotion
}

\author{
Authors: Alessandro Santuz ${ }^{1,2,3 *}$, Olivier Laflamme ${ }^{1}$, Turgay Akay ${ }^{1 *}$
}

${ }^{1}$ Atlantic Mobility Action Project, Brain Repair Centre, Department of Medical Neuroscience, Dalhousie University, Halifax, Nova Scotia B3H 4R2, Canada. ${ }^{2}$ Department of Training and Movement Sciences, Humboldt-Universität zu Berlin, 10115 Berlin, Germany. ${ }^{3}$ Berlin School of Movement Science, Humboldt-Universität zu Berlin, 10115 Berlin, Germany.

*Correspondence to: alessandro.santuz@dal.ca, turgay.akay@dal.ca

Abstract:

Safe locomotion relies on information from proprioceptors, sensory organs that communicate the position of body parts to the central nervous system. Proprioceptive circuits in the spinal cord are known to robustly regulate locomotion in challenging environments. The role of ascending pathways conveying proprioceptive information to the brain remains less clear. Through mouse genetic studies and in vivo electrophysiology, here we show that the systemic removal of proprioceptors leaves the animals in a constantly perturbed state, similar to that observed during mechanically perturbed locomotion in wild type. Yet, after surgical interruption of the ascending proprioceptive pathways, wild-type mice lose the ability to cope with external perturbations while walking. Our findings provide direct evidence of a pivotal role for ascending proprioceptive information in achieving safe locomotion.

\section{Main Text:}

Animals constantly move in complex environments. Locomotor actions often dictate the safe execution of challenging activities such as chasing prey, escaping danger, exploring new territories. In vertebrates, the generation of rhythmic and patterned activities such as those needed for locomotion is largely achieved through neuronal networks located in the spinal cord: the central pattern generators (CPGs) (1). The higher order integration of spatiotemporal information happening in the cerebral cortex is known to be important for initiating and halting locomotion, or regulating its speed (2-4). Yet, when somatosensory feedback is removed and supraspinal pathways are left intact, the motor commands generated by the CPGs alone are not sufficiently robust to allow for safe locomotion in the presence of external perturbations (5-7).

In mammals, information about body position in space and relative to the body itself(i.e., "proprioception") is conveyed by mechanosensory neurons found within muscles, tendons and joints (8). These neurons bear information from the muscle spindles, Golgi tendon organs (GTOs) and joints. While not much is known about the function of joint receptors in locomotion (8), feedback from muscle spindles (group Ia and II afferent fibers) and GTOs (group Ib) undoubtedly plays a role in mammalian locomotion $(6,9)$. In cats, mice, humans and other mammals, these proprioceptive afferents are pivotal for setting the timing of the gait cycle and facilitating the switch between different phases, such as propulsion and swing (3). The selective and systemic removal of proprioceptive afferents in mice can disrupt the locomotor patterns (10, 11) and the regulation of muscle activity needed to locomote at different speeds (12) or to cope with perturbations (7). Part of the proprioceptive information is elaborated locally at a spinal level (spinal sensory processing), but there are two main pathways that convey proprioceptive feedback from the hindlimb to supraspinal structures (supraspinal sensory processing): the dorsal spinocerebellar tract (originating from the Clarke's column) (13) and the dorsal column-medial 
lemniscus (DCML) pathway (8). Both have been shown to be major carriers of proprioceptive signals to the cerebellum (dorsal spinocerebellar tract) and to the somatosensory cortex via the brainstem (DCML). However, the importance of the role played by higher centers in integrating sensory information during locomotion is still obscure (5).

Here, we set out to explore how relevant is the supraspinal integration of proprioceptive feedback during murine locomotion, positing that not only spinal but also supraspinal sensory processing must be involved in the tuning of motor control when locomotion becomes challenging. During walking, we administered random mediolateral and anteroposterior perturbations to the animals, by means of sudden displacements or accelerations of the treadmill's belt. We used a combination of genetics, in vivo electrophysiology, spinal lesion models, and computational neuroscience to design a simple experiment that could decouple the contributions to sensory processing given by local (i.e., spinal) and supraspinal structures. By means of high-speed video recordings, electromyography (EMG) and an analysis framework based on linear and nonlinear tools, we addressed the role of supraspinal versus spinal processing of proprioceptive information during murine locomotion in challenging conditions.

First, we investigated the effects of external perturbations on locomotion in wild-type mice (Fig. 1). The perturbation protocol (Fig. S1) increased the cadence, or number of steps per minute (Fig. S6) and the step-to-step variability of the hip joint angle at touchdown, as shown by the Poincaré maps (Fig. 1A, Fig. S7). Additionally, perturbations reduced the knee- and anklejoint angle in the early stages of the swing phase, making these two joints less flexed than in normal, unperturbed locomotion (Fig. 1B). The root mean square values of the EMG signals $\left(\mathrm{RMS}_{\mathrm{EMG}}\right)$ recorded from the hindlimb were, on average, higher during perturbed locomotion (Fig. 1C, Fig. S8). To uncover the modular structure of muscle activations, we decomposed EMG activity into muscle synergies (Fig. S2) via non-negative matrix factorization $(14,15)$. Muscle synergies are represented by a set of time-invariant muscle weightings (or motor modules, describing the relative contribution of each muscle to a specific synergy) and a set of time-dependent activation patterns (or motor primitives). Three synergies were sufficient to reconstruct the original EMG signals of both unperturbed and perturbed walking (Fig. 1D). The obtained motor modules and motor primitives described three main phases of the gait cycle: 1) the weight acceptance, with the major contribution of knee extensors; 2) the propulsion, mostly involving the ankle extensors; 3) and the swing, characterized by the contribution of hip extensors and ankle flexors. During perturbed locomotion, compared to unperturbed walking, the timing of motor primitives did not undergo any noteworthy shift, as measured by the center of activity (Fig. 1D, Fig. S11). However, all primitives were longer active, as shown by the increased full width at half maximum (Fig. 1D, Fig. S10). The analysis of the local (Higuchi's fractal dimension) and global (Hurst exponent) complexity of motor primitives (Fig. 1E) showed that only the global complexity of these activation motifs was strongly affected by perturbations in wild-type mice (Fig. S12). The local complexity can be seen as a measure of "roughness" (or noise content) in the signal within each gait cycle, while the global complexity is a measure of how accurate each cycle's activations are, when compared to the others (Fig. S2D). Motor primitives were less accurate (i.e., higher Hurst exponent) when the animals were challenged by perturbations. These first observations in wild-type animals could show that perturbations to locomotion in intact animals elicited: a) higher variability and less flexion of joint angles; b) higher amplitude of EMG signals; and c) wider and less accurate motor primitives. 
bioRxiv preprint doi: https://doi.org/10.1101/2021.04.28.441796; this version posted April 29, 2021. The copyright holder for this preprint (which was not certified by peer review) is the author/funder, who has granted bioRxiv a license to display the preprint in perpetuity. It is made available under aCC-BY 4.0 International license.
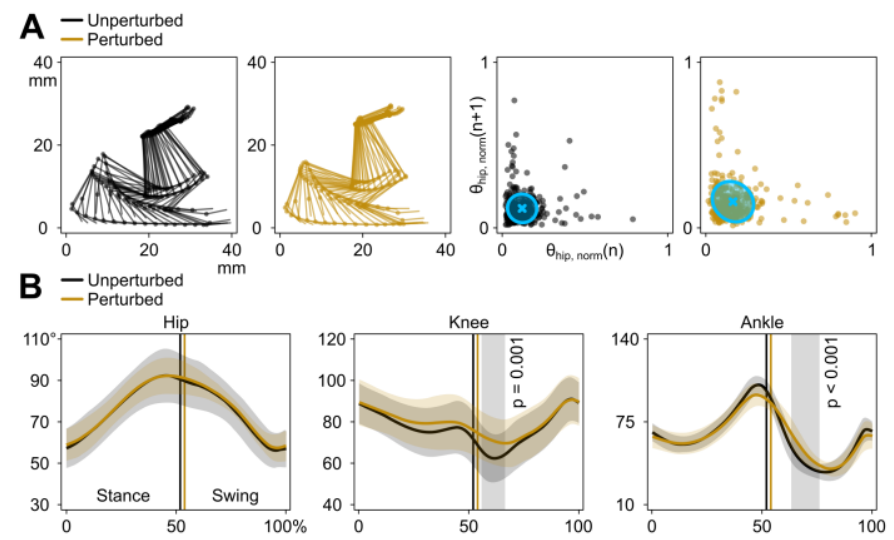

C 二 Unperturbed
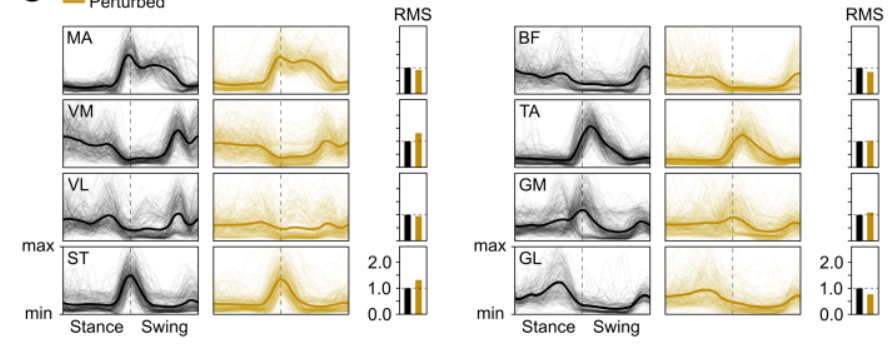

D 二 Unperturbed

Motor modules
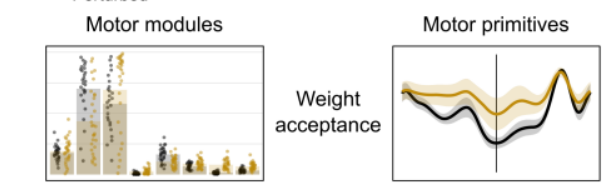

FWHM CoA
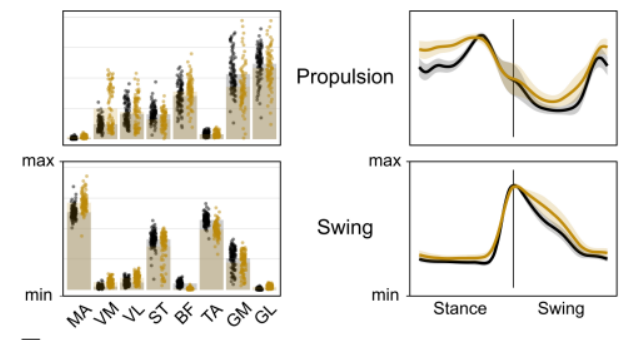

E
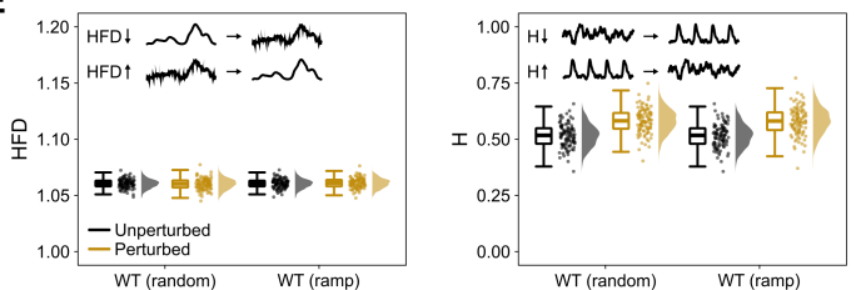

Fig. 1. Gait performance parameters of wild-type mice during unperturbed and perturbed locomotion. (A) Stick diagrams of one representative animal (left) and Poincaré maps (right) of the hip angle $\theta_{\text {hip }}$ at touchdown (n) and $(n+1)$ of all animals, with descriptor ellips e (see methods). (B) Average joint angles (thick lines) and standard deviation (shaded bands) for all animals. Vertical shaded areas denote differences detected by statistical parametric mapping. (C) Average electromyographic activity (thick lines) of the eight recorded muscles and individual trials (thin lines). RMS is normalized to unperturbed walking. Muscle abbreviations: $\mathrm{MA}=$ gluteus maximus, $\mathrm{VM}=$ vastus medialis, $\mathrm{VL}=v$. lateralis, $\mathrm{ST}=$ semitendinosus, $\mathrm{BF}=$ bicepsfemoris, $\mathrm{TA}=$ tibialis anterior, $\mathrm{GM}=$ gastrocnemius medialis, and $\mathrm{GL}=g$. lateralis.$(\mathrm{D})$ Motor modules and primitives of the three bootstrapped muscle synergies. Modules are presented on a normalized y-axis base. For simplifying the graphs, each point represents 10 nearest neighbors of the 1000 bootstrapped trials. For the motor primitives, the x-axis full scale represents the averaged step cycle (stance and swing normalized to the same amount of points) and the y-axis the normalized amplitude. Full width at half maximum (FWHM) and center of activity (CoA) of motor primitives are reported schematically (right). (E) Boxplots describing the Higuchi's fractal dimension (HFD or "roughness") and Hurst exponent (H or "accuracy") of the bootstrapped primitives for two perturbation protocols: random (Fig. S1) and one mediolateral displacement every $2 \mathrm{~s} \mathrm{(ramp).} \mathrm{Raw} \mathrm{data} \mathrm{points} \mathrm{(each} \mathrm{point} \mathrm{represents} 10$ nearest neighbors of the 1000 bootstrapped trials) and their density estimates are presented to the right side of each boxplot. 
Then, we set out to study the aftermath of genetic removal of one class of proprioceptors: the muscle spindles. We used Egr $3^{-/-}$mice, a model in which muscle spindles regress after birth. In those mutant animals, the number of steps per minute was higher than in wild type, while the swing phase was shorter (Fig. S6). Moreover, in this group the kinematics were generally not affected by the presence of external perturbations, but the variability of hip joint angle at touchdown was higher than in wild type (Fig. 2A and B, Fig. S7). Contrary to wild-type animals, $\mathrm{Egr}^{-/-}$did not increase the $\mathrm{RMS}_{\mathrm{EMG}}$ when exposed to perturbations (Fig. 2C, Fig. S8). Yet, they showed increased duration of the weight acceptance and swing primitives when compared to their wild-type littermates, and the main activity shifted later in time in the swing primitive (Fig. 2D, Fig. S9,Fig. S10, Fig. S11). When exposed to perturbations though, the mutants only produced a shorter and earlier activation motif in the muscle synergy for the weight acceptance (Fig. 2D, Fig. S9,Fig. S10, Fig. S11). As in wild-type animals, the roughness (i.e., the local complexity) of motor primitives was not affected by perturbations and was not different from that of intact controls (Fig. 2E, Fig. S12). However, the activation patterns in both normal and perturbed locomotion were as accurate (i.e., same global complexity) as those of perturbed locomotion in wild type (Fig. 2E, Fig. S12). This indicates an inherently disrupted control of locomotion due to the systemic lack of feedback from muscle spindles, independently on the external stimuli. In short, this second part of the experiment revealed that the systemic lack of muscle spindles produced: a) increased variability of kinematics; b) the inability to modulate the hindlimb kinematics or EMG amplitude in the presence of perturbations; and c) wider, less accurate (i.e., globally less complex) motor primitives when compared to wild type, with again no nuances when comparing unperturbed and perturbed walking. 
bioRxiv preprint doi: https://doi.org/10.1101/2021.04.28.441796; this version posted April 29, 2021. The copyright holder for this preprint (which was not certified by peer review) is the author/funder, who has granted bioRxiv a license to display the preprint in perpetuity. It is made available under aCC-BY 4.0 International license.

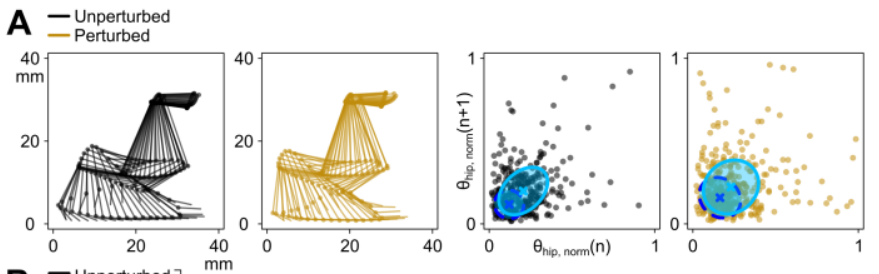

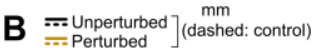
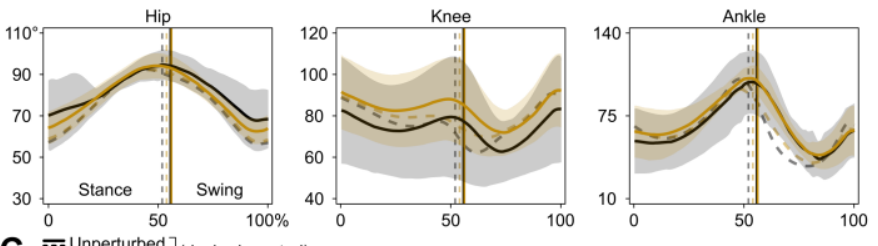

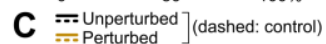
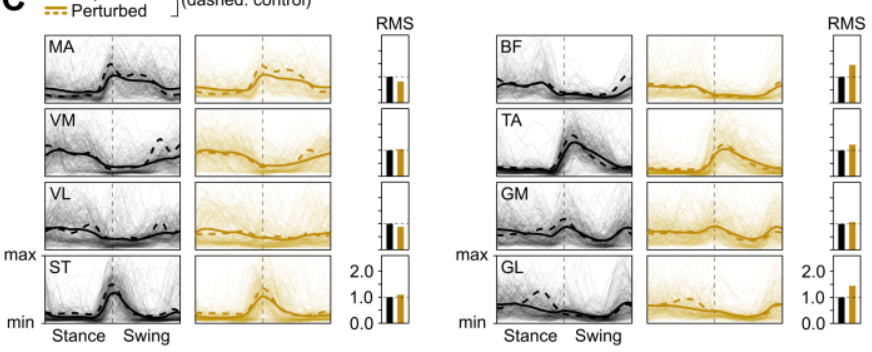

D $\underset{\text { F.: Unperturbed }}{\text { Pertured }}$ (dashed: control)

Motor modules
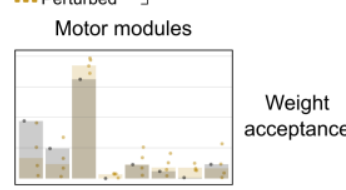

Motor primitives
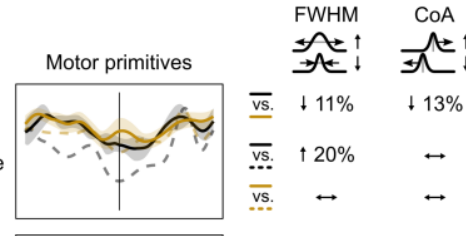

ક્ષ!

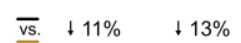

vs. $\downarrow 11 \%+13 \%$

$\begin{array}{ccc}\overline{\text { vs. }} & \uparrow 20 \% & \leftrightarrow \\ \overline{\text { vs. }} & \leftrightarrow & \leftrightarrow\end{array}$
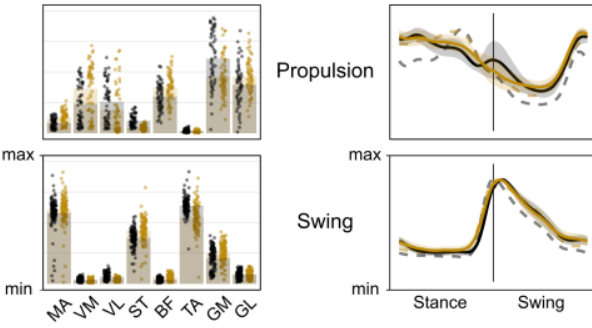

ㄷ. $\leftrightarrow \quad \leftrightarrow$

$\begin{array}{lll}\frac{\mathrm{vs} .}{\mathrm{vs}} & \leftrightarrow & \leftrightarrow \\ \mathrm{v} & & \leftrightarrow\end{array}$

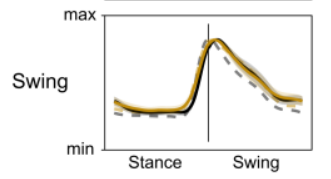

E
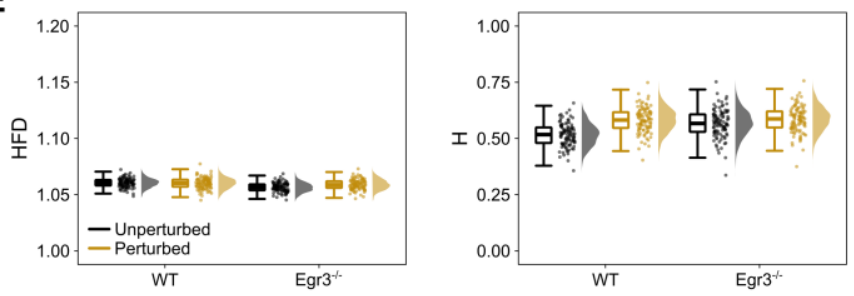

Fig. 2. Gait performance parameters of Egr $3^{-/}$mice during unperturbed and perturbed locomotion. (A) The descriptor ellipses in the Poincaré maps are overlaid to those of wild type (Fig. 1). (B to D) Control is wild type. The RMS in (B) is normalized to unperturbed walking of Egr $3^{-/-}$ . See caption of Fig. 1 for all other details. 
The genetic removal of muscle spindles can however raise the question of whether the animals could adapt to the partial lack of sensory feedback during the first weeks of life. Moreover, GTOs are known to be important players in the control of locomotion, but the Egr $3^{-/-}$ model only targets muscle spindles. To tackle these two potential issues, we acutely ablated both muscle spindles and GTOs in adult mice by systemic injection of diphtheria toxin in PV Cre::Avil ${ }^{\text {iDTR }}$ mice (Fig. S3). Before injection, these mice behaved largely similar to wild-type animals (Fig. S13). After injection however, they underwent major degradation of sensory neurons, resulting in severely disrupted kinematics, independently on the administered perturbations (Fig. 3A and B). Specifically, the step-to-step variability of the hip joint angle at touchdown increased dramatically as compared to the pre-injection recordings (Fig. S7) and the ankle joint was less flexed throughout almost all of the stance phase (Fig. 3B). Moreover, there was a big effect of sensory ablation on the stance and swing time variability (Fig. S5) and duration (Fig. S6). The $\mathrm{RMS}_{\mathrm{EMG}}$ was on average higher and unaffected by perturbations after injection (Fig. 3C, Fig. S8). While the number and function of muscle synergies (Fig. 3D) were not influenced by acute sensory ablation, the timing of motor primitives was indeed. When comparing pre- and post-injection recordings, we detected a main effect on the timing of main activation that was shifted to the right in the propulsion synergy, with all the remaining main effects having small size and interactions showing large effect size in all synergies for both duration and main peak activity, a sign of cross-over interaction or opposite effects in the two groups (Fig. 3D, Fig. S9, Fig. S10, Fig. S11). This showed yet again that the systemic disruption of proprioceptive pathways leads to the inability to modulate control's robustness depending on external demands. A consideration that was confirmed by the fractal analysis of motor primitives: similar to what we observed in Egr $3^{-/-}$, the now acute disruption of feedback from proprioceptors made the activation patterns less accurate (i.e., higher Hurst exponent), once more making unperturbed and perturbed locomotion numerically indistinguishable (Fig. 3E, Fig. S12). Somewhat interestingly though, the sensory ablation was followed by a large increase of the roughness of motor primitives (Fig. 3E, Fig. S12). However, this might be due to the known effect of speed on the Higuchi's fractal dimension (16): after injection, the animals could not cope with pre-injection speeds and had to be tested at half those speeds $(0.15$ instead of 0.25 and $0.30 \mathrm{~m} / \mathrm{s}$ ), also resulting in longer cycle times and lower cadence (Fig. S5, Fig. S6). In summary, the acute ablation of both muscle spindles and GTOs induced: a) increased kinematic variability and EMG amplitude and the inability to modulate them in the presence of perturbations; and $b$ ) rougher and less accurate motor primitives, with widespread reorganization of timing. 
bioRxiv preprint doi: https://doi.org/10.1101/2021.04.28.441796; this version posted April 29, 2021. The copyright holder for this preprint (which was not certified by peer review) is the author/funder, who has granted bioRxiv a license to display the preprint in perpetuity. It is made available under aCC-BY 4.0 International license.

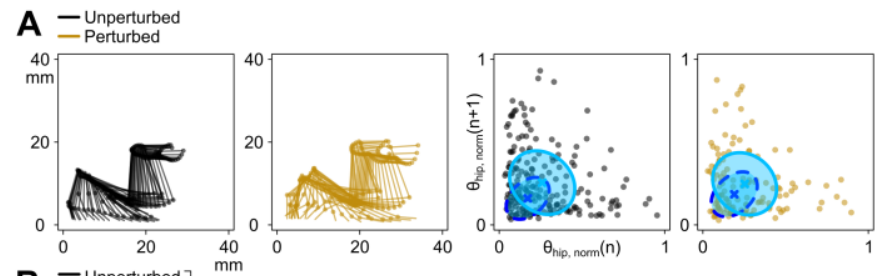

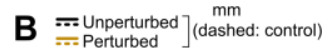
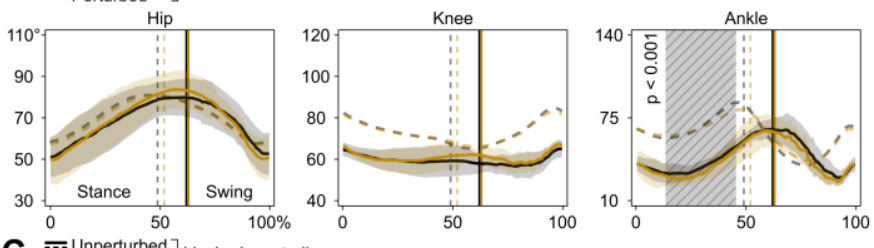

C $\stackrel{=}{=}$ Unperturbed $]$ (dashed: control)
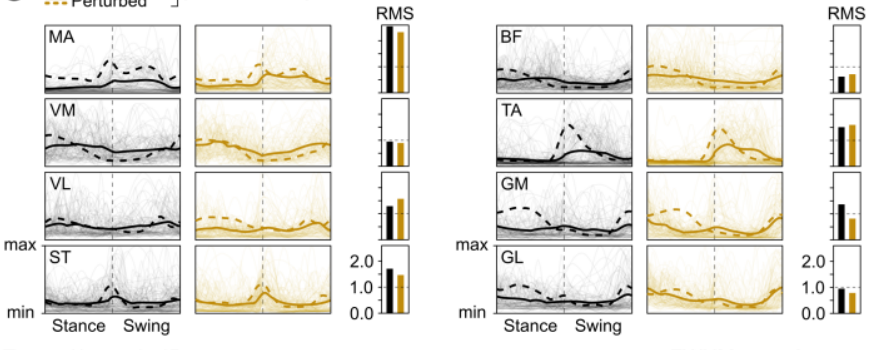

D $\underset{\text { F.: Unperturbed }}{\text { Perbed }}$ (dashed: control)

Motor modules
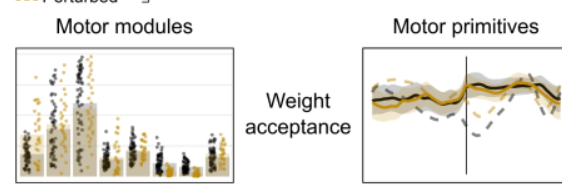

FWHM COA
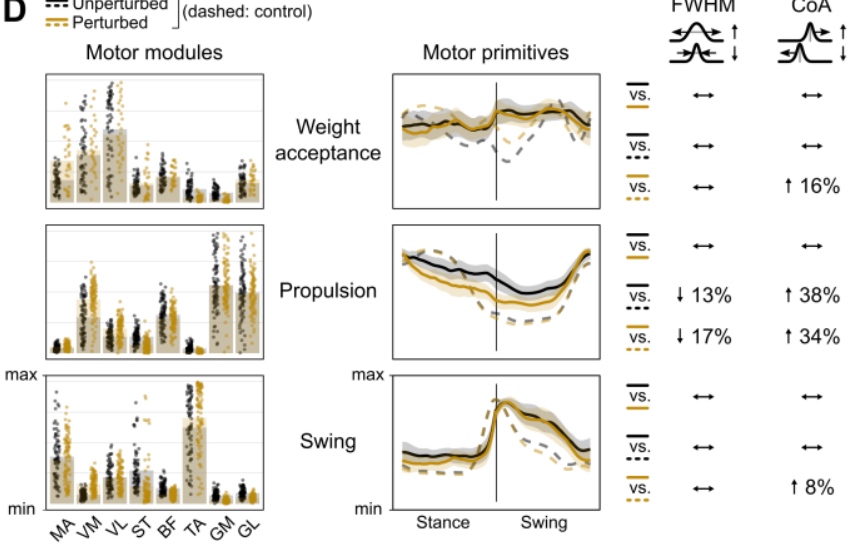

E
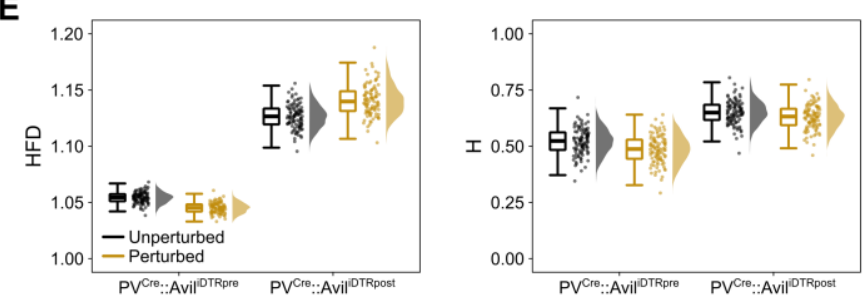

Fig. 3. Gait performance parameters of $\mathbf{P V}^{\mathrm{Cre}}$ ::Avil ${ }^{\mathrm{iDTR}}$ mice during unperturbed and perturbed locomotion. (A) The descriptor ellipses in the Poincaré maps are overlaid to those of pre-DTX injection recordings. (B to D) Control is pre-injection. The RMS in (B) is normalized to unperturbed walking recorded pre-injection. See caption of Fig. 1 for all other details. 
Nevertheless, the neurophysiological changes genetically induced in $\mathrm{Egr} 3^{-/-}$and $\mathrm{PV}^{\mathrm{Cre}}:$ :Avil ${ }^{\mathrm{iDTR}}$ animals were affecting both the spinal as well as the ascending proprioceptive information conduction, not allowing to make any consideration on the specific contribution of supraspinal proprioceptive integration to locomotion. To overcome this last hurdle in our experimental design, we surgically lesioned the DCML pathway in wild-type mice (Fig. S4). This lesion would disrupt sensory information transfer to the brain centers including the cortex through the thalamic relay (17) and partly to the cerebellum through the dorsal spinocerebellar pathway (13). After lesion, the animals did not show big changes in either the kinematics or EMG activity when compared to pre-lesion recordings (Fig. 4A, B and C, Fig. S5, Fig. S6, Fig. S7, Fig. S8). The number and function of synergies was not affected by the lesion either (Fig. 4D). Yet, the timing of motor primitives underwent interesting modifications. First, in lesioned animals there was a negligible effect of perturbations on both the duration (Fig. S9) and the main activity timing (Fig. S11). Moreover, the motor primitives during both unperturbed and perturbed locomotion were similarly accurate as those of unperturbed walking in intact animals (Fig. 4E, Fig. S12). In summary, with this last part we could show that interrupting the flow of proprioceptive information to the brain in wild-type animals caused the loss of ability to interact with perturbations with minimal changes of the measured parameters in unperturbed locomotion. A conclusion supported also by the behavioral observation that three out of five animals would not manage sudden accelerations of the treadmill's belt, interrupting locomotion immediately after the stimulus was administered.

In conclusion, we showed that the systemic removal of proprioceptors in mice resulted in impaired locomotion proportionally to the level of ablation (i.e., of only muscle spindles or of both spindles and GTOs). This shows that sensory information from proprioceptors is needed to robustly cope with external perturbations and that the lack of sensory feedback has direct

25 implications on the animal's behavior, negatively affecting the kinematics and making muscle activation patterns less accurate and adaptable. Moreover, the surgical interruption of ascending pathways carrying proprioceptive information to the brain through the spinal cord compromised the ability of wild-type mice to deal with perturbations: a demonstration that supraspinal integration of sensory information from proprioceptors is crucial to robustly control locomotion 30 at need. 
bioRxiv preprint doi: https://doi.org/10.1101/2021.04.28.441796; this version posted April 29, 2021. The copyright holder for this preprint (which was not certified by peer review) is the author/funder, who has granted bioRxiv a license to display the preprint in perpetuity. It is made available under aCC-BY 4.0 International license.

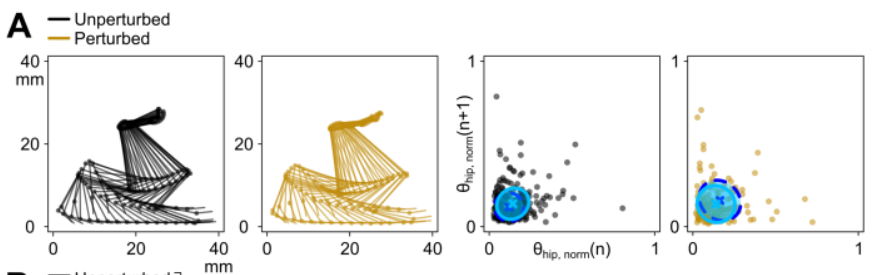

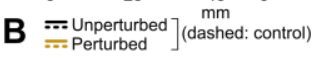
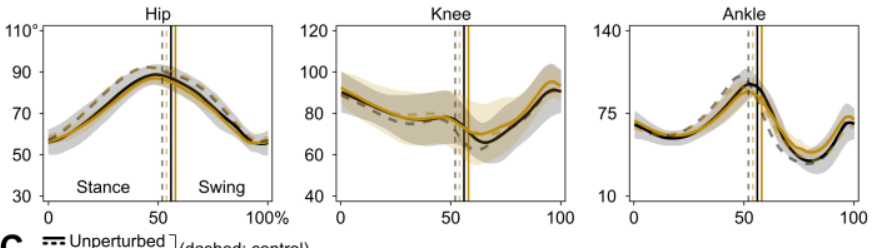

C. $\underset{\cdots}{\text {.... Unperturbed }}]$ (dashed: control)
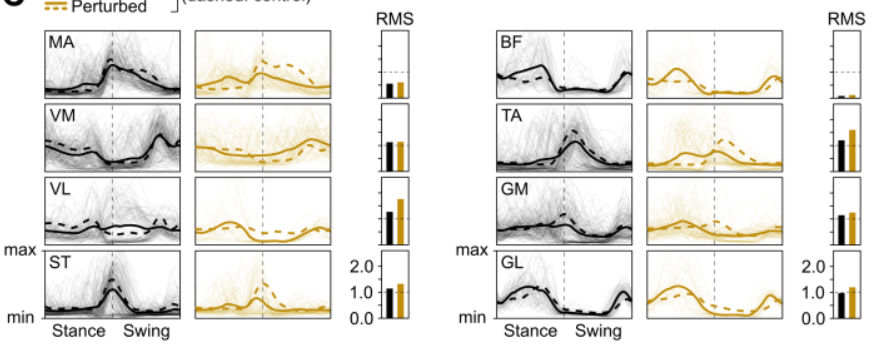

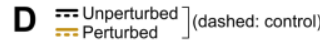

Motor modules
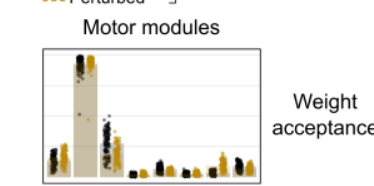

Motor primitives

FWHM CoA
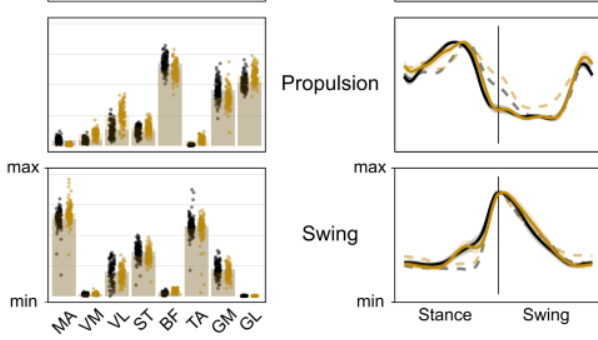

武 牙!

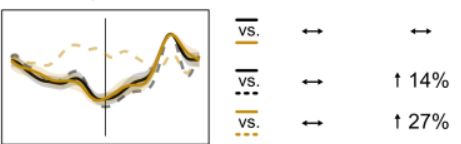

$\mathbf{E}$
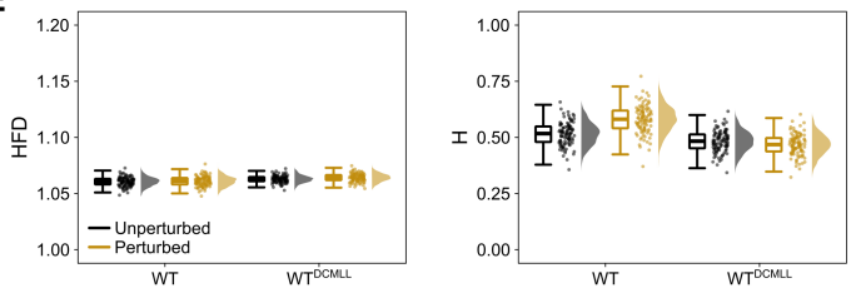

Fig. 4. Gait performance parameters of wild-type mice during unperturbed and perturbed locomotion after DCML pathway lesion. (A) The descriptor ellipses in the Poincare maps are overlaid to those of wild type, pre-lesion (Fig. 1). (B to D) Control is pre-lesion. The RMS in (B) is normalized to unperturbed walking recorded pre-lesion (Fig. 1). See caption of Fig. 1 for all other details. 


\section{Materials and methods}

$\underline{\text { Mouse lines }}$

All procedures were performed according to the guidelines of the Canadian Council on Animal Care and approved by the local councils on animal care of Dalhousie University with protocols \#19-014 and \#19-021. Recordings were conducted on 17 adult (age $89 \pm 26$ days; mass $24.4 \pm 2.9 \mathrm{~g}$ ) male mice on C57BL/6 background fed ad libitum. Seven C57BL/6 wild-type mice were used for the DCML lesion protocol. To investigate the role of muscle spindles, we performed experiments with five Egr $3^{-/-}$mice $(18)$. To investigate the role of proprioceptive feedback from muscle spindles and Golgi Tendon Organs, we have used three PV ${ }^{\mathrm{Cr}}::$ Avil ${ }^{\text {iDTR }}$ and two PV Pre::Avil ${ }^{\text {iDTR }:: \text { Rosa }^{\text {EGFP }} \text { mice (11). Three PVCre::Rosa }}{ }^{\text {EGFP }}$ mice were used for the sham experiments (Fig. S14). For the recordings, mice of different litters but same genotype were allocated to the relevant experimental group using as the only inclusion criteria: a) the genotyping, b) a minimum mass of $22 \mathrm{~g}$ and c) a minimum age of 50 days. The mice used to breed the experimental mice were obtained from: PV Cre: Jackson Laboratories (017320) (19);

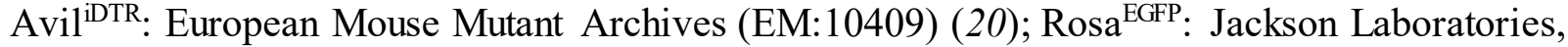
USA (010701) (21); Egr3 ${ }^{-/}$: courtesy of Dr. Warren Tourtellotte (Northwestern University, Chicago, IL; currently Cendar Cinai Medical Center, Los Angeles, CA); Wild type: littermates of different breeding colonies in the lab with C57/BL6 background.

\section{$\underline{\text { EMG implantation surgeries }}$}

20 Each mouse received an electrode implantation surgery as previously described $(7,10,22)$. Eight bipolar EMG electrodes were implanted $(23,24)$ to as many muscles of the right hind limb. The muscles that were implanted were: gluteus maximus (MA), vastus medialis (VM), vastus lateralis (VL), semitendinosus (ST), biceps femoris (BF), tibialis anterior (TA), gastrocnemius medialis (GM) and gastrocnemius lateralis (GL). Briefly, mice were anesthetized with isoflurane at $2-3 \%$ concentration through inhalation at $11 / \mathrm{min}$ and ophthalmic eye ointment was applied to the eyes. The skin was sterilized by using a three-part skin scrub by means of Hibitane (Chlorhexidine gluconate 4\%), alcohol and povidone-iodine. Two small midline skin incisions on the back and one on the posterior part of the right hind leg were made to expose the target muscles and position the custom 3D-printed back-mounted connector cap. The electrodes were then drawn from the most anterior neck incision to the leg incisions subcutaneously and the EMG connector cap was sutured to the skin. Afterwards, each electrode was implanted in the muscles as described before (23), the incisions were closed, anesthetic was discontinued and buprenorphine $(0.03 \mathrm{mg} / \mathrm{kg})$ and ketoprofen $(5.00 \mathrm{mg} / \mathrm{kg})$ were injected subcutaneously. The mice were then placed in a heated cage for at least six days and returned to their regular mouse rack once recovered. Food mash and hydrogel were provided for the first three days after the surgery multiple times a day and subsequently once a day until the animal returned to the rack. Handling of the mice was mostly avoided until they were fully recovered and at least for one week. Additional injections of buprenorphine and ketoprofen were performed in 24-and 12-hour intervals, respectively, for at least 48 hours after surgery.

\section{$40 \quad$ Spinal lesion surgeries}

The DCML pathway lesion surgeries that were conducted after the first EMG recordings followed the same anesthesia and care protocols as the EMG implantation surgeries. After preparation and anesthesia, one midline skin incision of around $10 \mathrm{~mm}$ was made to expose the back muscles that were then separated with a scalpel to reveal the 7th or 8th thoracic vertebral body (25). A laminectomy was then performed, the dura mater opened and the spinal cord 
lesioned at the 9th (three mice) or 10th (two mice) thoracic spinal segment using tungsten steel microscissors. Muscles were then stitched over the missing spinous process using absorbable suture, the skin closed and the animals placed in their recovery cage for at least three days.

\section{Diphtheria toxin (DTX) delivery}

Intraperitoneal DTX (D0654 lyophilized, Sigma-Aldrich, St. Louis, MO, United States) injection was carried out on three PVCre::AviliDTR and two PVCre.:Avil ${ }^{\text {iDTR.::Rosa }}{ }^{\text {EGFP }}$ experimental mice and on PVCre:: Rosa ${ }^{\text {EGFP }}$ mice for the sham experiments. We injected each mouse with $100 \mu \mathrm{g} / \mathrm{kg}$ of toxin (11) diluted in ultrapure water (Millipore, TMS-006-C) and let the animals in a confined cage for three days, when they could return to their rack. Recordings were conducted on the seventh day after injection.

$\underline{\text { Immunohistochemistry }}$

To assess the success of the DCML pathway lesion at the 9th/10th spinal segment, we perfused the animals immediately after euthanasia. After thoracotomy, the mice were perfused with $20 \mathrm{ml}$ of saline solution followed by $10 \mathrm{ml}$ of $4 \%$ paraformaldehyde solution (PFA) through the left cardiac ventricle. The spinal cord was dissected and immersed in PFA for at least $24 \mathrm{~h}$. The 4th lumbar and 1st thoracic spinal segments were then dissected and cryoprotected by immersion in $30 \%$ sucrose-PBS solution for at least $24 \mathrm{~h}$ or until sunk. After cryoprotection, the spinal cord sections were embedded in optimal cutting temperature (OCT) mounting medium, flash frozen on dry ice and stored at $-80^{\circ} \mathrm{C}$. The tissue was then sectioned transversally at $30 \mu \mathrm{m}$ by means of a cryostat (Leica CM3050 S, Leica Biosystems AG, Muttenz, Switzerland) and placed on microscope slides to dry for at least one hour. Half of the sections were then prepared for Toluidine Blue O (TBO) staining and the remaining half for Protein Kinase C Gamma (PKCG) staining. For TBO (T3260 Sigma-Aldrich, St. Louis, MO, United States) staining, slides were washed three times for five minutes in 1xPBS to remove OCT and then immersed in 1:1000 TBO-1XPBS solution for other five minutes. Slides were then washed once for five minutes in $1 \times$ PBS and mounted on coverslips using PermaFluor Aqueous mounting medium (Thermo Fisher Scientific, Waltham, MA, United States). For PKCG staining of the corticospinal tract, after the three initial washes in 1xPBS, the slides were incubated for 60 minutes in 5\% blocking solution ( $5 \%$ BSA in $1 \times$ PBS $+0.3 \%$ Triton) at room temperature. Subsequently, the sections were incubated overnight at $4{ }^{\circ} \mathrm{C}$ in a primary antibody solution consisting of 1:1000 rabbit-antiPKCG (Abcam ab71558 rabbitpAB to PKCG, Abcam, Cambridge, UK) in 1\% blocking solution ( $1 \%$ BSA in $1 \times$ PBS $+0.3 \%$ Triton). The next day, the tissue received three 10 -minute washes in 1xPBS and a secondary incubation of 90 minutes at room temperature in 1:500 Invitrogen A32794 donkey-anti-rabbit alexa fluor 555 plus (Invitrogen, Carlsbad, CA, United States) in 1\% blocking solution. After other three 10-minute washes in 1xPBS, slides were mounted on coverslips. To assess the success of proprioceptive afferent ablation in $\mathrm{PV}^{\mathrm{Cre}}::$ Avil ${ }^{\mathrm{DTR}}$ mutants after DTX injection, we dissected the dorsal root ganglia and spinal cord at the 4th lumbar spinal segment after perfusion. Following the procedures reported above, we prepared the tissue for Hoechst 33342 staining (both dorsal root ganglia and spinal cord), RUNX3 staining (dorsal root ganglia) and VGLUT1 staining (spinal cord). The procedure for Hoechst 33342 (Abcam ab228551) staining was identical to that described above for HBO staining. RUNX3 and VGLUT1 staining protocols were identical to that for PKCG, with different primary and secondary antibody incubations. For RUNX3 (Jessell laboratory), we incubated overnight at $4{ }^{\circ} \mathrm{C}$ with 1:5000 guinea pig-anti RUNX3 in 1\% blocking solution and then for 45 minutes at room temperature with 1:1000 goat anti-guinea pig alexa fluor 555 plus (Invitrogen). For VGLUT1 (Jessell laboratory), we incubated overnight at $4{ }^{\circ} \mathrm{C}$ with 1:8000 rabbit-anti VGLUT1 in 1\% 
blocking solution and then for 60 minutes at room temperature with 1:500 goat anti-rabbit alexa fluor 555 plus (Invitrogen).

\section{$\underline{\text { EMG }} \underline{\text { recordings }}$}

After recovery from surgery, all animals walked on a custom-built treadmill (workshop of the Zoological Institute, University of Cologne, Germany) capable to administer perturbations through quick mediolateral displacements and accelerations of the belt. We recorded at least 30, often non-consecutive, gait cycles from each animal during unperturbed and perturbed walking. Each animal walked at two speeds: 0.25 and $0.30 \mathrm{~m} / \mathrm{s}$. In the case of severely compromised locomotor patterns, such as in the PV Cre::Avil ${ }^{\text {iDTR }}$ mutants after DTX injection, the speed was reduced to $0.15 \mathrm{~m} / \mathrm{s}$ to let the animals cope with the belt's speed. Perturbations were administered at random time intervals (minimum $0 \mathrm{~ms}$, maximum $1200 \mathrm{~ms}$ after the previous perturbation was completed) and consisted of either a) a sudden acceleration of the belt that doubled the speed and then returned to the initial one in $200 \mathrm{~ms}$ (100 ms acceleration, $100 \mathrm{~ms}$ deceleration) or b) a mediolateral motion that suddenly displaced the whole treadmill by $6 \mathrm{~mm}$ in $100 \mathrm{~ms}$ with constant velocity. The randomized perturbation protocol included 20 anteroposterior and 20 mediolateral perturbations, randomly distributed along $30 \mathrm{~s}$ (Fig. S1); the cycle started at random points and would repeat itself automatically if needed. A less challenging protocol, including only one mediolateral perturbation every $2 \mathrm{~s}$, was administered to those animals that could not cope with the random protocol. Namely, this happened for the WT after DCML lesion. This reduced protocol produced similar effects to the randomized protocol on the gait spatiotemporal parameters (Fig. S5) and is referred to in the figures as "ramp". EMG signals were recorded by connecting the electrodes via the EMG connector to an amplifier (MA 102, workshop of the Zoological Institute, University of Cologne, Germany) and recorded at a sampling frequency of $10 \mathrm{kHz}$. Animals were euthanized immediately after the last recording with a high dose of sodium pentobarbital $(100 \mathrm{mg} / \mathrm{kg})$ through intraperitoneal injection.

\section{$\underline{\text { Kinematics }}$}

The gait cycle segmentation (paw touchdown and lift-off timing) was obtained by the elaboration of high-speed videos. The kinematics of the hindlimb were acquired with a highspeed camera operating at $500 \mathrm{~Hz}$ with resolution 1280 x 800 pixels (IL3, Fastec Ima ging, San Diego, CA, United States). For markerless body part tracking we used DeepLabCut (26) v2.1.7. We labelled 16 landmarks on 297 frames taken from 30 videos of 15 different animals assigning the $95 \%$ of those images to the training set without cropping. Namely, we labelled six calibration markers, iliac crest and hip (highlighted by two white dots placed with an oil-based marker under brief $3 \%$ isoflurane anesthesia through inhalation at $11 / \mathrm{min}$ ), knee, ankle, fifth metatarsal, toe tip of the rear paw, toe tip of the front paw and the four paw reflections on the mirror placed under the treadmill at $45^{\circ}$ angle. We used a ResNet-50-based neural network $(27,28)$ with default parameters for 1030000 training iterations and two refinements of 100000 and 300000 iterations, respectively. We validated with one shuffle and found the test error was 2.75 pixels and the train error 2.57 pixels.

40 Of the 16 landmarks, we used eight for the segmentation of the gait cycle: the six calibration, the metatarsal and the rear toe tip markers. Following a procedure extensively reported previously $(22,29)$, we processed the data to detect touchdown and lift-off of the rightside hindlimb. For touchdown estimation, we used the modified foot contact algorithm developed by Maiwald and colleagues (30). For estimating lift-off, we used the paw acceleration 45 and jerk algorithm $(22,29)$. We found [LOe $-20 \mathrm{~ms}$, LOe $+20 \mathrm{~ms}$ ] to be the sufficiently narrow interval needed to make the initial lift-off estimation. Both approaches have been validated 
against a set of 104 manually-labelled touchdown/lift-off events from 4 videos of two animals at different speeds $(0.2$ and $0.3 \mathrm{~m} / \mathrm{s})$ and showed a true error within the frame rate of the camera (i.e., $\leq 2 \mathrm{~ms}$ ).

Poincaré maps were produced by mapping the hip-joint angle $\theta_{\text {hip,norm }}$, normalized between the minimum and the maximum of each gait cycle, at touchdown of the gait cycle $n(x-$ axis) and at touchdown of the following gait cycle (y-axis). To quantify the dispersion of the obtained map, we used the descriptors (31) SD1 and SD2, which are calculated as follows:

$$
\left\{\begin{array}{c}
S D 1=S D\left[\theta_{\text {hip }, \text { norm }}(n)-\theta_{\text {hip,norm }}(n+1)\right] \cdot \sqrt{2} / 2 \\
S D 2=\sqrt{2 \cdot S D\left[\theta_{\text {hip,norm }}(n)\right]^{2}-0.5 \cdot S D\left[\theta_{\text {hip,norm }}(n)-\theta_{\text {hip,norm }}(n+1)\right]^{2}}
\end{array}\right.
$$

\section{$\underline{\text { EMG bootstrap }}$}

Given the challenges imposed by the implantation of many muscles (eight), some EMG channels suffered degradation of the signal between different sessions or right after surgery. In order to avoid issues in the extraction of muscle synergies originating from missing data, we used a bootstrap-like resampling approach reported before (7). This allowed us to substantially reduce the number of needed animals, in accordance with the principles of humane animal research. Briefly, for each group and condition, we created 1000 resampled data sets each containing eight-muscle EMG data for 30 gait cycles. Each muscle activity in every gait cycle was randomly selected, without replacement, from all the available recorded activities for that group of animals and condition. In the case of perturbed locomotion, we followed the random perturbation protocol and inserted a perturbation every five steps or less (choosing randomly) and picking each time from the relevant data set (i.e., if the selected perturbation was a mediolateral displacement to the left, we would only pick from the EMG data sets that contained a mediolateral perturbation to the left for that cycle, etc.). We so obtained 1000 trials of 30 gait cycles each for WT, unperturbed walking, pre-DCML pathway lesion; another 1000 for Egr $3^{-/}$, perturbed walking; and so on for a total of 12000 trials, given the 12 analyzed conditions.

Muscle synergies extraction

Muscle synergies data were extracted from the bootstrapped EMG activity through a custom script (R v4.0.4, R Core Team, 2021, R Foundation for Statistical Computing, Vienna, Austria) using the classical Gaussian non-negative matrix factorization (NMF) algorithm as extensively reported in the past $(15,29,32,33)$. A schematic representation of the procedure can be found in Fig. S2. The source code for the pre-processing of raw EMG data and for the extraction and classification of muscle synergies is available at https://github.com/alesantuz/musclesyneRgies (34). Version 1.1.0, used in this paper, is archived at Zenodo (DOI: 10.5281/zenodo.4724766).

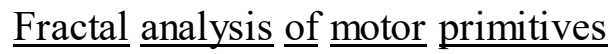

To assess the local and global complexity of the bootstrapped motor primitives, we calculated the Higuchi's fractal dimension (HFD) and Hurst exponent (H), respectively $(35,36)$. The numerical procedures for obtaining both metrics were recently reported in detail (22). HFD was calculated with a maximum window size of 10 points. $H$ was calculated following the rescaled range (R/S) approach (37) with a minimum window length of 200 points. HFD values range from 1 to 2 , with increasing values correlating to increasingly complex data and HFD $=1.5$ indicating random Gaussian noise $(35,38,39)$. $\mathrm{H}$ can vary between 0 and 1 . For $0.5<\mathrm{H}<1$, in 
the long-term high values in the time series (the motor primitive in our case) will be probably followed by other high values and a positive or negative trend is visible $(40,41)$. For $0<\mathrm{H}<$ 0.5 , in the long term high values in the series will be probably followed by low values, with a frequent switch between high and low values $(40,41)$. A completely random series will have $\mathrm{H}=$ $0.5(41,42)$. In other words, values of $\mathrm{H}$ approaching 0.5 from both ends indicate more complex (or random) behavior of the time series (36).

\section{Sham experiments}

Three PV ${ }^{\mathrm{Cre}}:: \mathrm{R}_{\mathrm{S} a}{ }^{\mathrm{EGFP}}$ mice underwent electrode implantation as described above. After recovery, the same recording protocol used for the other mice was performed. Then, the three animals were injected with DTX (Fig. S14) and after seven days another recording session was completed. Finally, the mice underwent the same surgery as the five lesioned animals, only this time without the actual lesion. The last recording session was then performed after recovery from surgery and the animals were perfused and dissected.

\section{$\underline{\text { Statistics }}$}

To investigate the effects of perturbations and sensory ablation on the kinematics, gait spatiotemporal parameters, EMG activity and muscle synergy-related metrics, we followeda Bayesian multilevel modeling approach implemented in the R package brms 2.15.0 (43, 44). For each variable of interest, we built a mixed effects model containing both "fixed" and "random" effects. The constant effects analyzed were the locomotion condition (i.e., walking with or without perturbations), the animal group (with pre/post conditions where relevant) and their interaction. In all groups, we added the random effects as a by-animal varying intercept. Each model was run with five independent Markov chains of 10000 iterations, with the first 4000 warm-up iterations used only for calibration and then discarded, thus resulting in 30000 post-warm-up samples. Convergence of the chains and sufficient sampling of posterior distributions were confirmed by ensuring a potential scale reduction factor $\hat{\mathrm{R}}<1.01$ and an effective sample size of at least $20 \%$ of the number of iterations. We so obtained the $95 \%$ credible intervals, defined as those intervals around the point estimates having a 95\% probability of encompassing the population value, given the data and the prior assumptions (45). We used different priors depending on the investigated parameters and based on the mean and two standard deviations of previously recorded data. Specifically, we used normal priors with the following means and standard deviations: stance duration $\sim \mathrm{N}(115 \mathrm{~ms}, 55 \mathrm{~ms})$; swing duration $\sim \mathrm{N}(95 \mathrm{~ms}, 30 \mathrm{~ms})$; cadence $\sim$ $\mathrm{N}(625$ steps/min, 160 steps/min); Poincaré descriptors $\sim \mathrm{N}(0.2,0.2) ; \mathrm{FWHM} \sim \mathrm{N}(65,17)$; CoA $\mathrm{N}(85,30) ; \mathrm{H} \sim \mathrm{N}(0.55,0.15)$. HFD values often produce target distributions having features with a resolution that cannot be grasped by the Hamiltonian model used by brms. Due to this, we used flat priors for HFD to avoid getting biased estimates. Since the EMG-related parameters (i.e., $\mathrm{RMS}_{\mathrm{EMG}}, \mathrm{FWHM}$, CoA, HFD and $\mathrm{H}$ ) were obtained by resampling of the EMG signals as described above, the mixed effects models for these variables were obtained by sampling without replacement ten random values from the 1000 obtained with the bootstrap-like procedure. This was done in order to calculate the posterior distributions based on samples having the same size

40 as the number of animals (five, two trials each) assigned to each group. Moreover, hindlimb joint angles were compared by evaluating the one-dimensional statistical parametric mapping (46). All calculations were performed in $\mathrm{R}$ v4.0.4.

\section{Data availability}

The datasets generated and analyzed during the current study and an exhaustive explanation of their structure are available at the Zenodo repository (DOI: 10.5281/zenodo.4724766). 
bioRxiv preprint doi: https://doi.org/10.1101/2021.04.28.441796; this version posted April 29, 2021. The copyright holder for this preprint (which was not certified by peer review) is the author/funder, who has granted bioRxiv a license to display the preprint in perpetuity. It is made available under aCC-BY 4.0 International license.
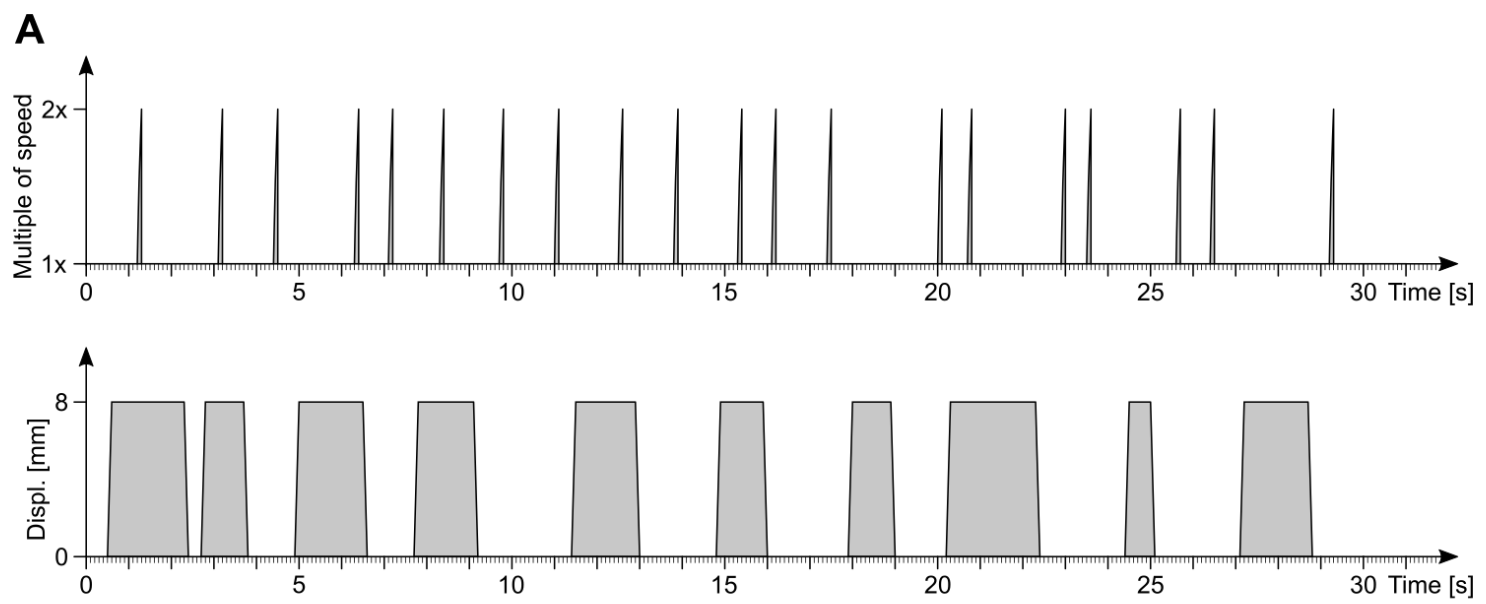

B
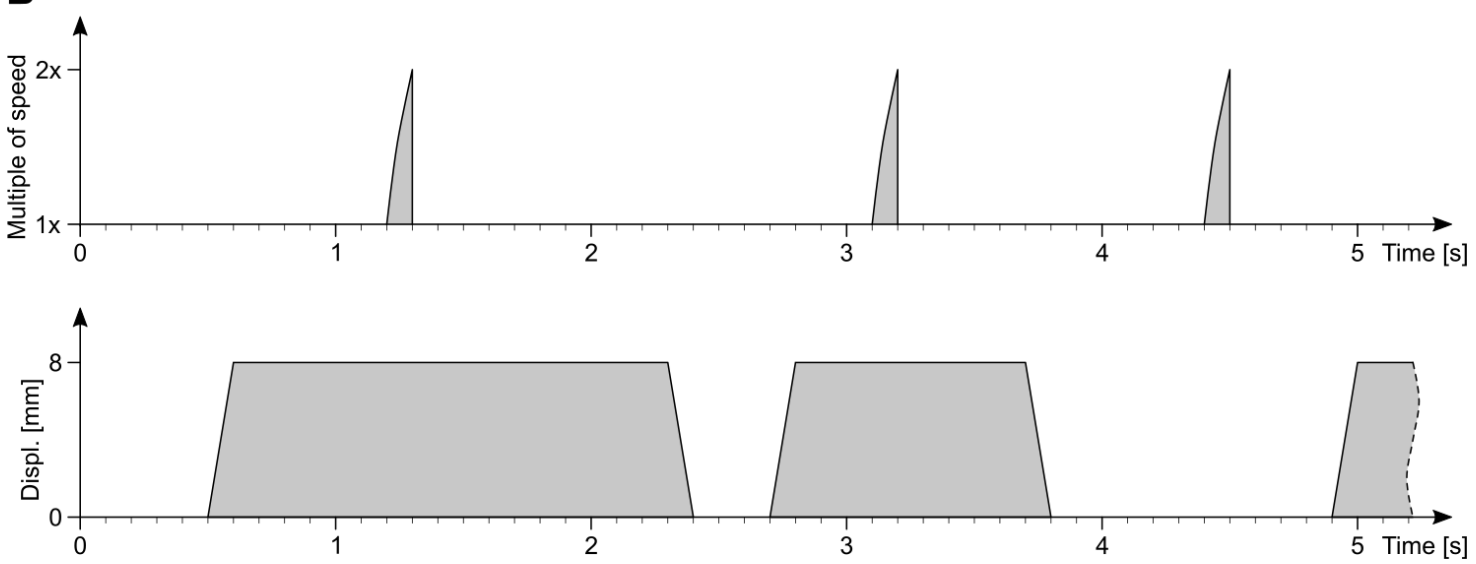

Fig. S1. Schematic representation of the perturbation protocol. (A) The whole protocol of $30 \mathrm{~s}$ that would repeat itself indefinitely at need. Sudden accelerations (top) and mediolateral displacements (bottom) of the belt are presented as shaded areas. (B) Zoom in the first $5.2 \mathrm{~s}$. 
bioRxiv preprint doi: https://doi.org/10.1101/2021.04.28.441796; this version posted April 29, 2021. The copyright holder for this preprint (which

was not certified by peer review) is the author/funder, who has granted bioRxiv a license to display the preprint in perpetuity. It is made available under aCC-BY 4.0 International license.

A

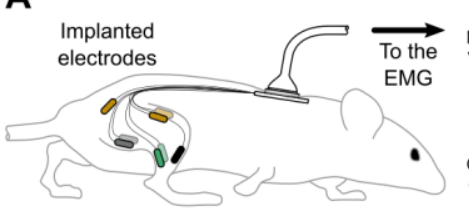

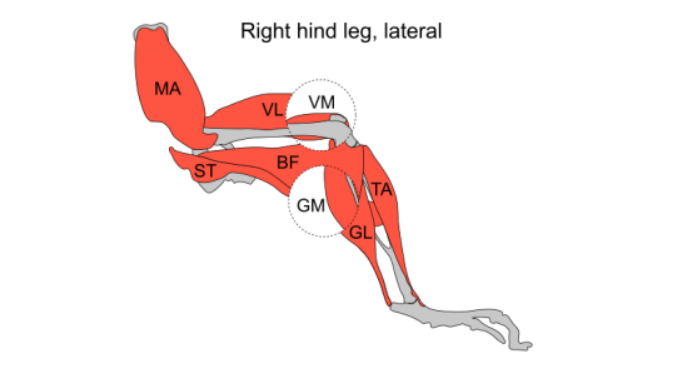

\section{B}

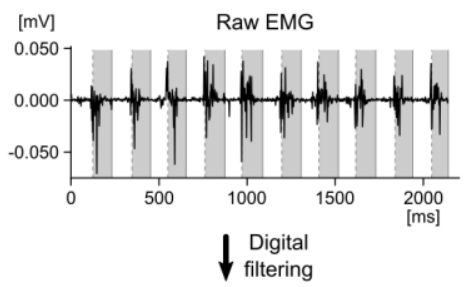

Filtered, time-normalized EMG

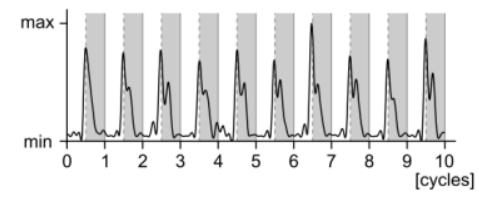

C
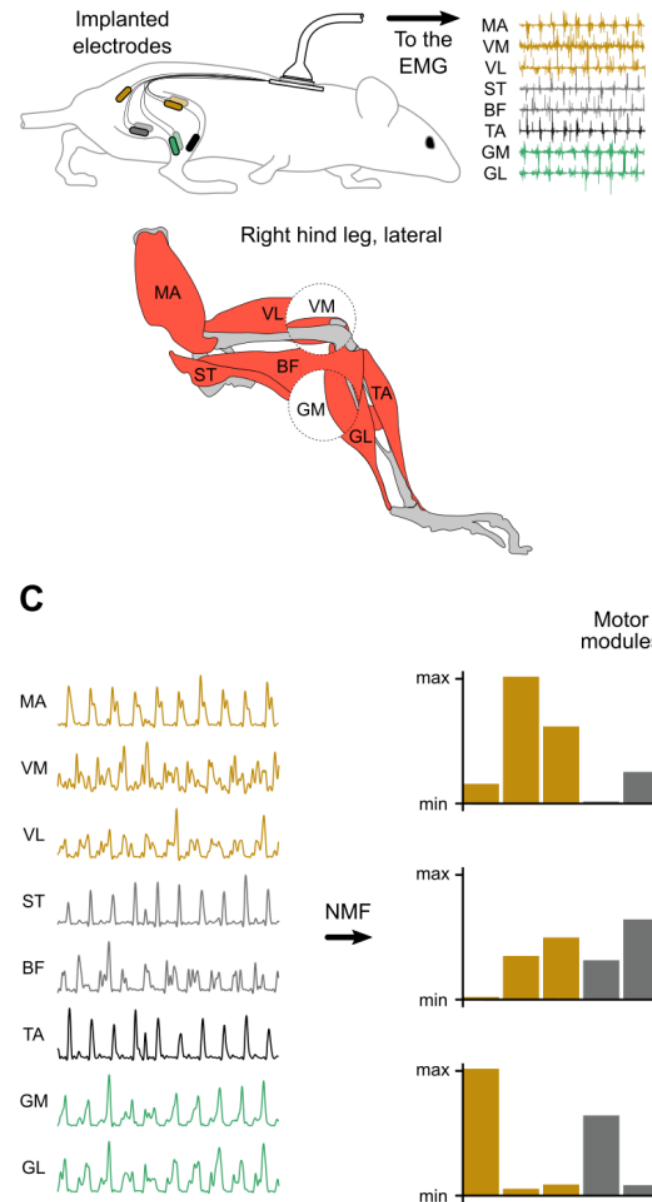

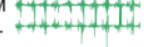

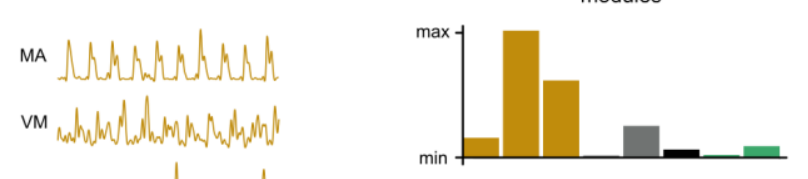

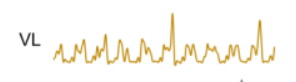

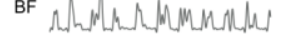
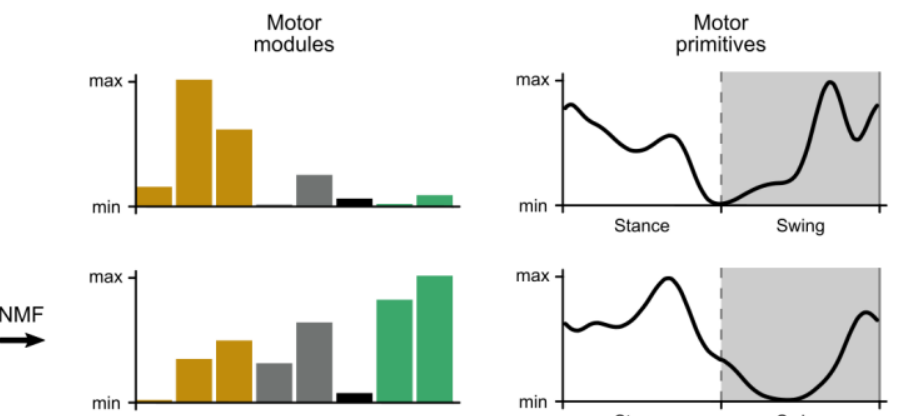

D
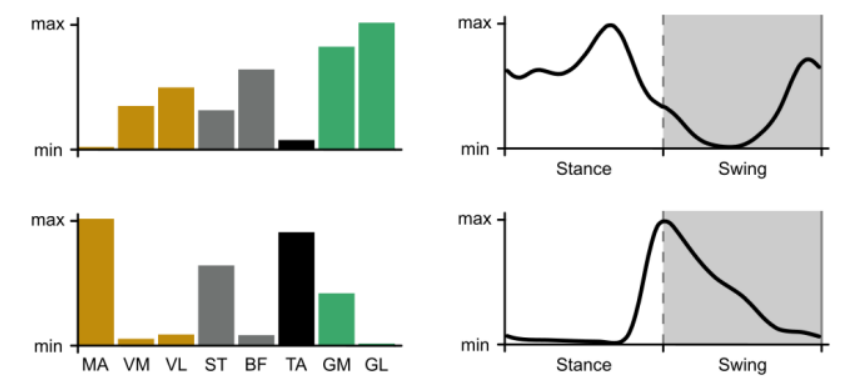

Cycle A (smoother than B)

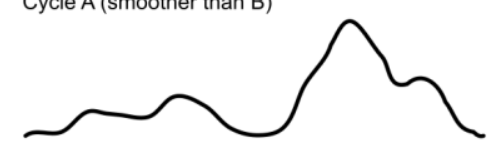

Cycle B (rougher than A)

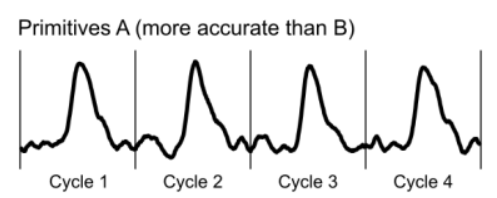

Primitives B (less accurate than A)
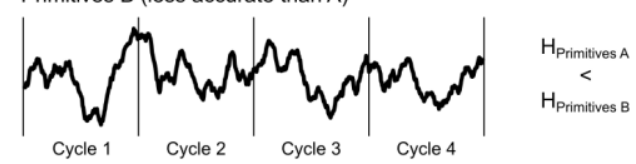

Fig. S2. Schematic representation of the muscle synergies approach and following fractal analysis. (A) Eight muscles were implanted in the right hindlimb. (B) Raw EMG was filtered and time- and amplitude-normalized. (C) Filtered and normalized EMG was factorized with nonnegative matrix factorization and classified into motor modules (time-invariant muscle weightings) and motor primitives (time-dependent synergistic coefficients). (D) Interpretation of the two metrics employed to carry out the fractal analysis of motor primitives. The Higuchi's fractal dimension (HFD, top row) is a measure of local complexity and it increases together with the "roughness" of motor primitives at a single cycle level (thus the term "local"). The Hurst exponent (H, bottom row) is a measure of global complexity and it decreases if the "accuracy" of motor primitives decreases across several gait cycles (thus the term "global"). 

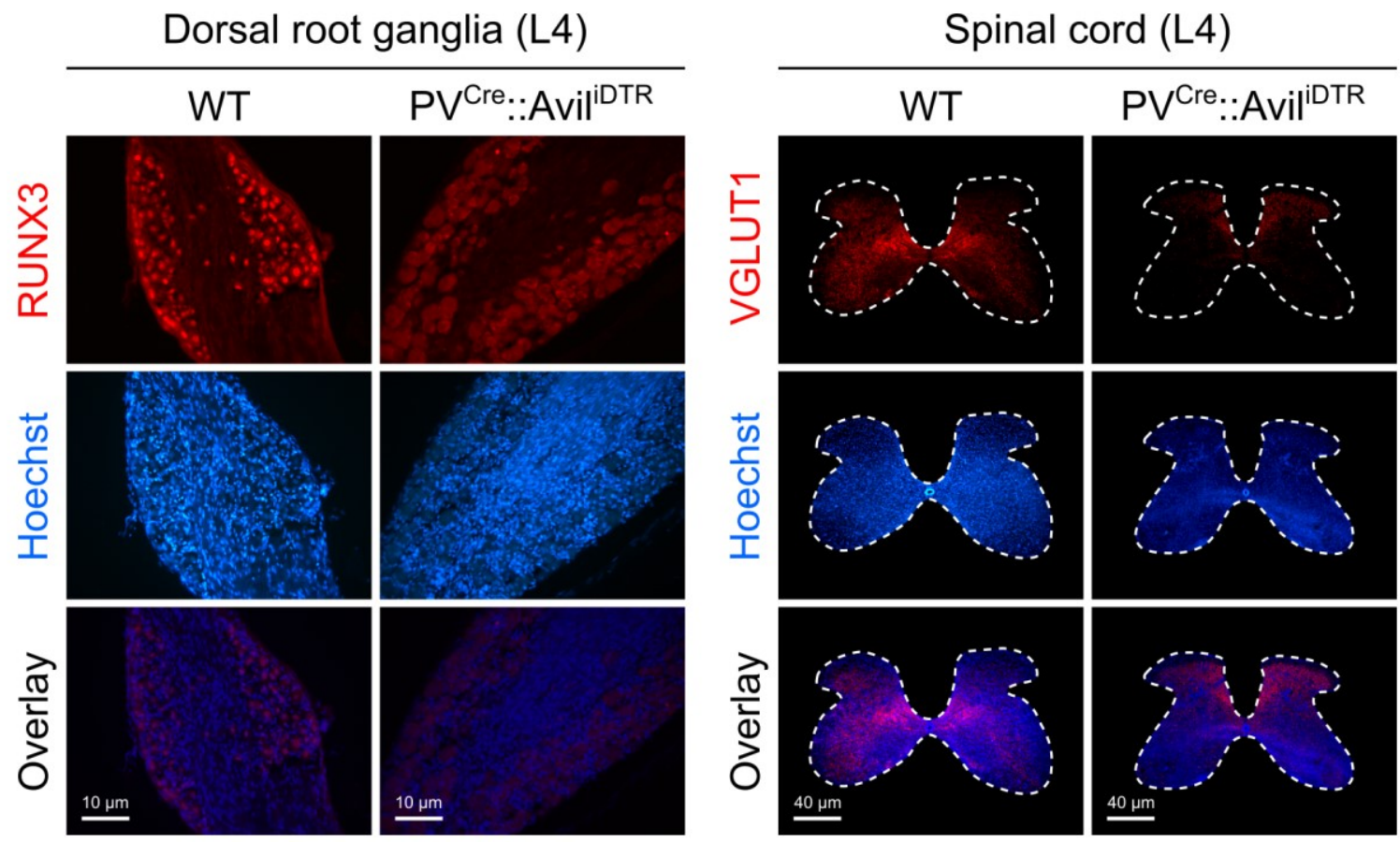

Fig. S3. Acute ablation of proprioceptive afferents with diphtheria toxin (DTX). Dorsal root ganglia (left) and spinal cord (right) sections taken at the $4^{\text {th }}$ spinal segment in wild-type (WT) and mutant ( PV $^{\mathrm{Cre}}::$ :Avil ${ }^{\text {iDTR }}$ ) mice. Runt-related transcription factor 3 (RUNX3) is

selectively expressed in intact proprioceptive sensory neurons located in dorsal root ganglia. Vesicular glutamate transporter 1 (VGLUT1) is expressed in central sensory terminals. After DTX injection in PV ${ }^{\mathrm{Cre}}:$ :Avil ${ }^{\mathrm{DTR}}$ mice, the proprioceptive neuron terminals are eliminated at all spinal levels, leaving the fewer in number corticospinal and non-proprioceptive sensory terminals unaffected. 
bioRxiv preprint doi: https://doi.org/10.1101/2021.04.28.441796; this version posted April 29, 2021. The copyright holder for this preprint (which was not certified by peer review) is the author/funder, who has granted bioRxiv a license to display the preprint in perpetuity. It is made available under aCC-BY 4.0 International license.

A

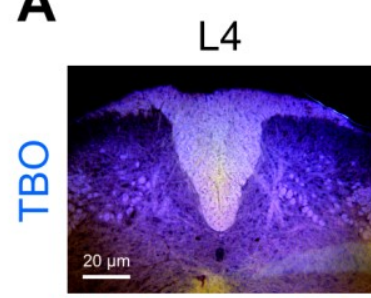

$\mathrm{T} 1$
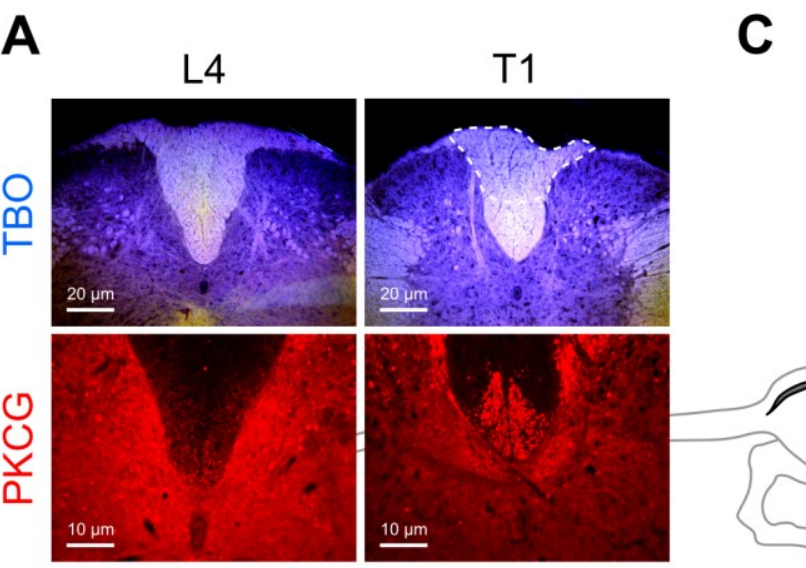

B
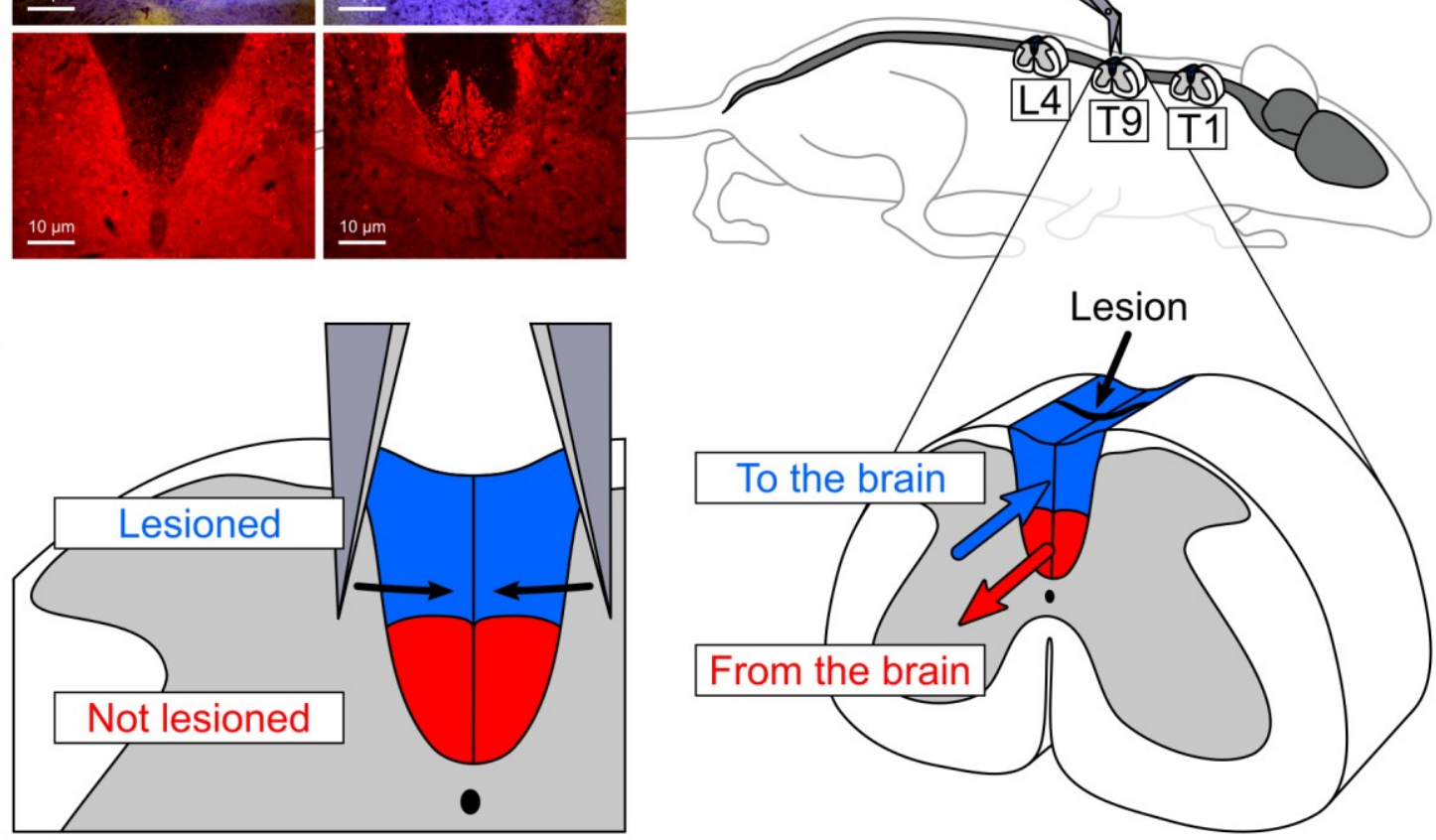

Fig. S4. Lesion of the dorsal column-me dial lemniscus (DCML) pathway leaves corticospinal tract intact. (A) After toluidine blue (TBO) staining, lesioned afferent axons result in damaged tissue rostral (spinal segment T1) but not caudal (L4) with respect to the lesion. Protein kinase $\mathrm{C}$ gamma (PKCG) is expressed in the axons of the corticospinal tract (CST) and can be used to evaluate the integrity of the latter after surgery. (B) The surgery was aimed at the lesion of the DCML (most dorsal portion of the dorsal column), leaving the CST (most ventral portion of the dorsal column) intact. (C) The lesion was carried out at the $9^{\text {th }}$ thoracic spinal segment. Sections at L4 and T1 were used to evaluate the success of the surgery. 
bioRxiv preprint doi: https://doi.org/10.1101/2021.04.28.441796; this version posted April 29, 2021. The copyright holder for this preprint (which was not certified by peer review) is the author/funder, who has granted bioRxiv a license to display the preprint in perpetuity. It is made available under aCC-BY 4.0 International license.

A
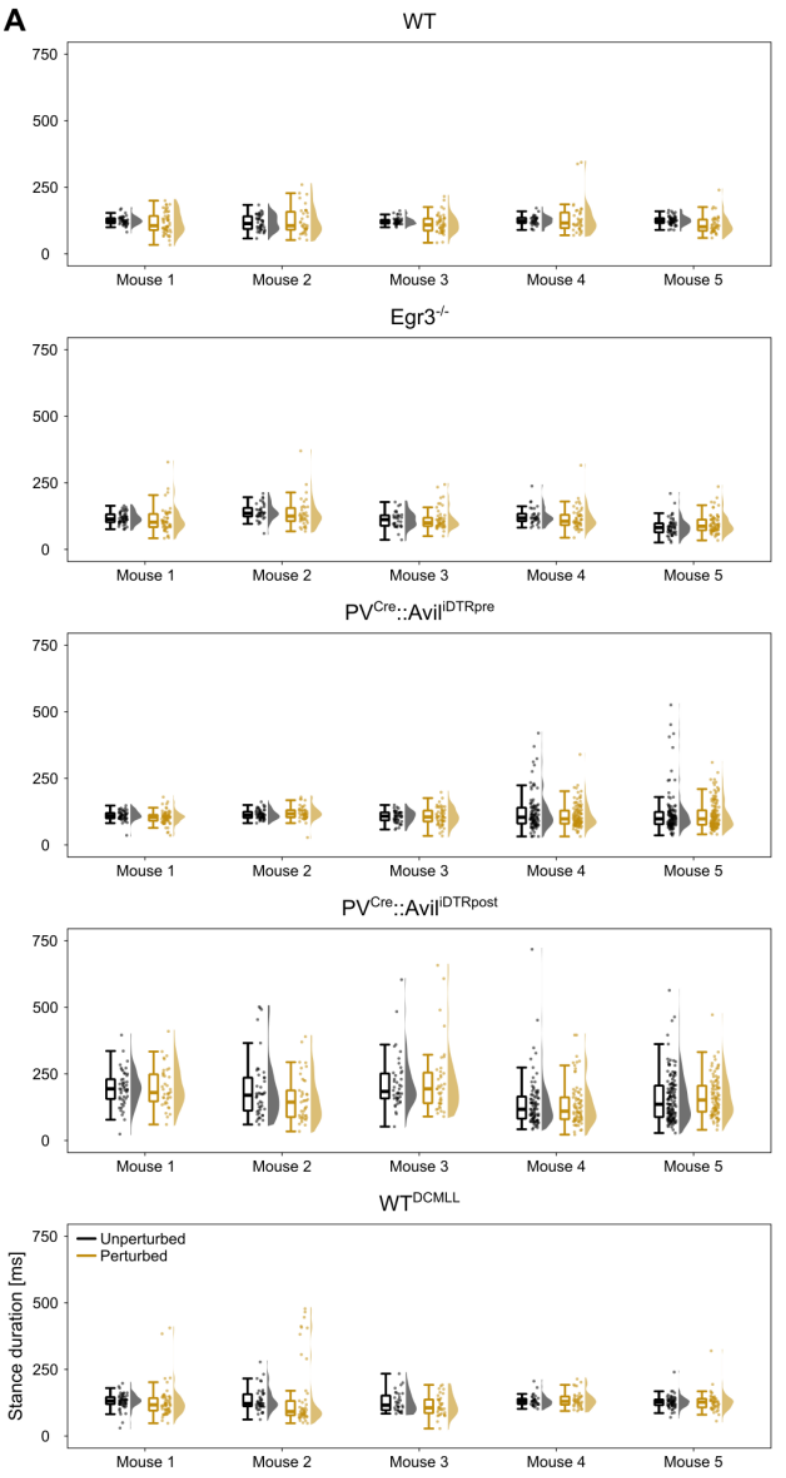

B $\quad$ WT
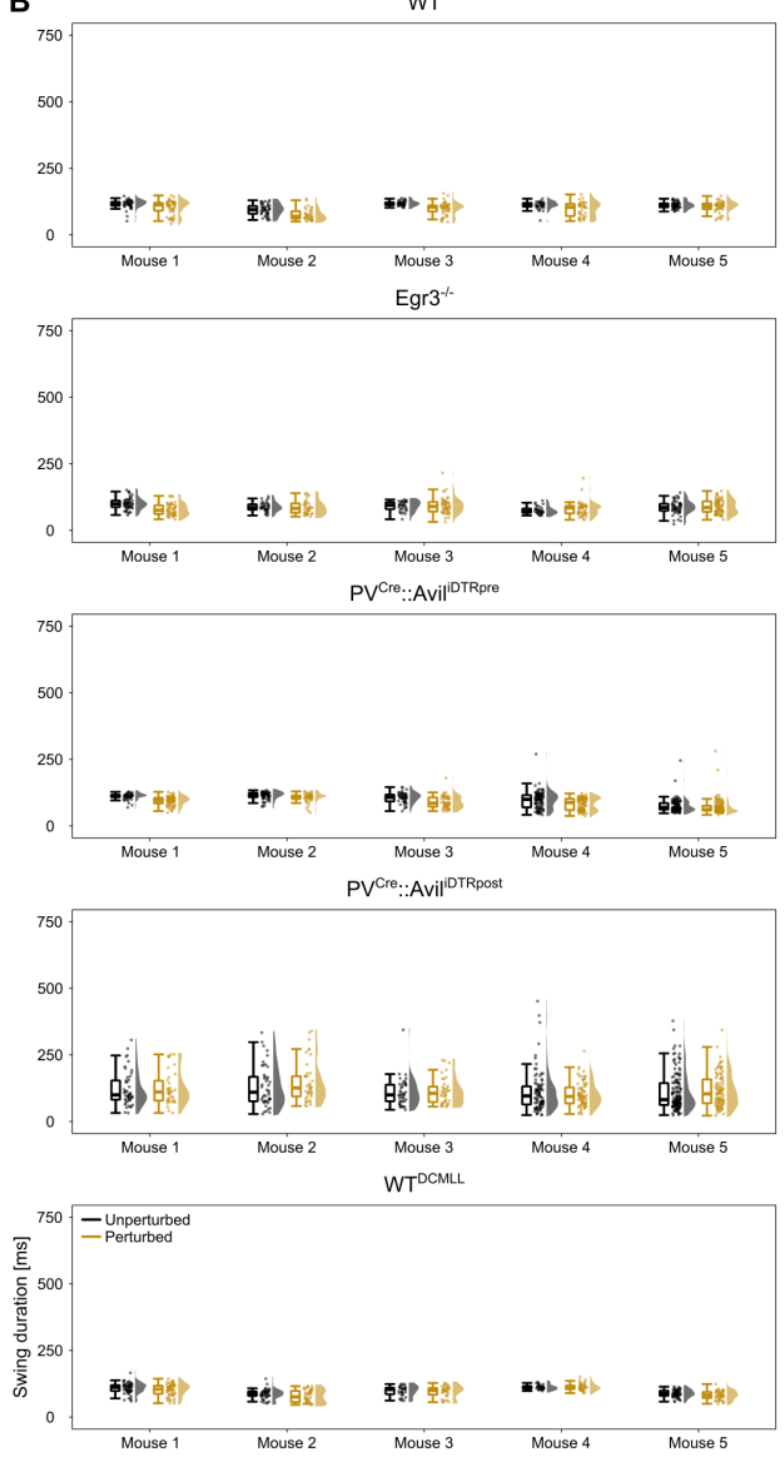

Fig. S5. Gait temporal parameters. Stance (A) and swing (B) phase duration for each step recorded in every animal. Individual step values, pictured as dots, and their distributions are shown next to the relevant boxplot. 
bioRxiv preprint doi: https://doi.org/10.1101/2021.04.28.441796; this version posted April 29, 2021. The copyright holder for this preprint (which was not certified by peer review) is the author/funder, who has granted bioRxiv a license to display the preprint in perpetuity. It is made available under aCC-BY 4.0 International license.

A
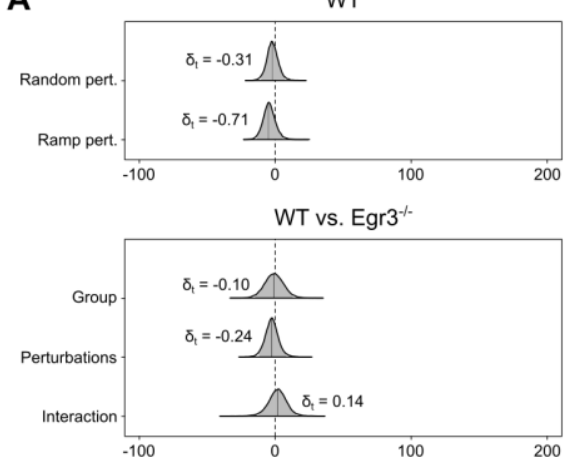

PgV Cre.:Avil ${ }^{\text {iDTRpre }}$ vs. PV Cre.::Avil ${ }^{\text {iDTRpost }}$
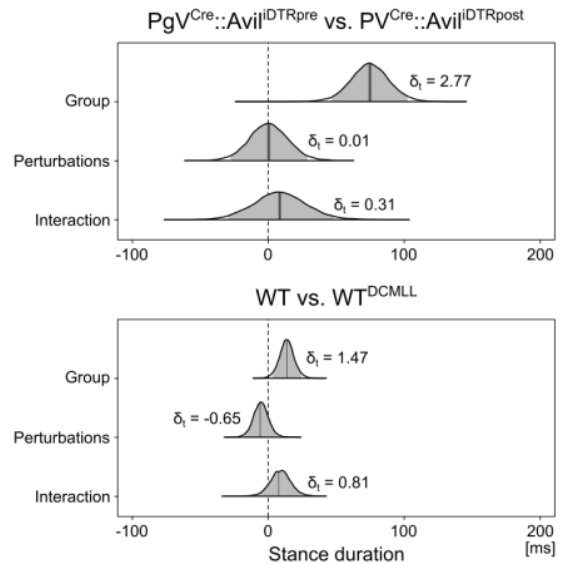

B

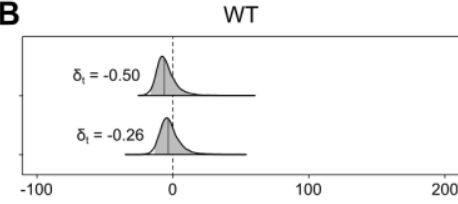

WT vs. Egr3 ${ }^{-1}$

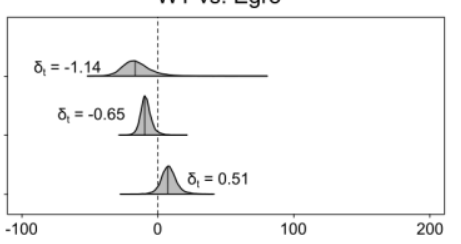

PgV Cre.:Avil $^{\text {iDTRpre }}$ vs. PV Cre.:Avil ${ }^{\text {iDTRpost }}$

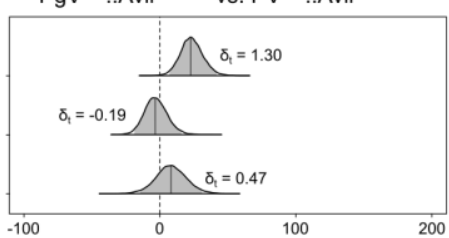

WT vs. WT DCMLL.

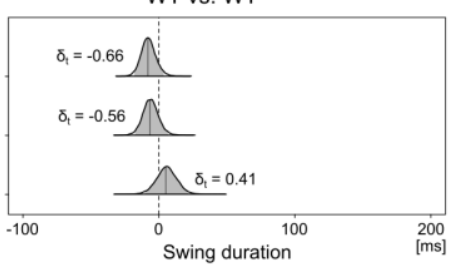

C

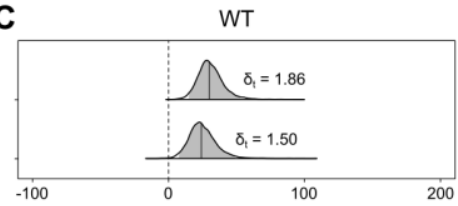

WT vs. Egr3 ${ }^{-1-}$

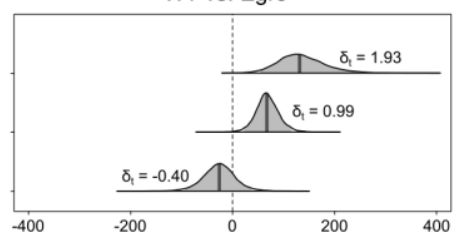

PgV Cre.:Avil ${ }^{\text {iDTRpre }}$ vs. PV Cre.:Avili ${ }^{\text {IDTpost }}$

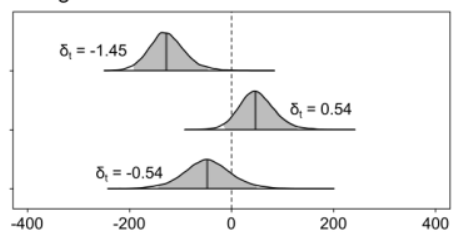

WT vs. WT ${ }^{\text {DCMLL }}$

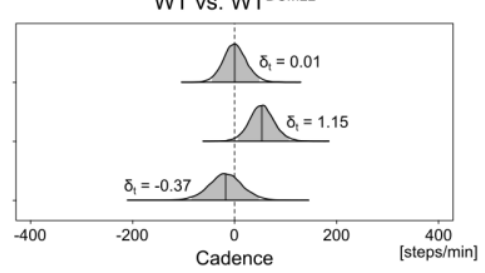

Fig. S6. Posterior $\mathbf{9 5 \%}$ credible intervals for the estimated differences in gait temporal parameters. (A) The $95 \%$ credible intervals and their probability distributions (shaded areas) describe the effects and interaction of the investigated groups and perturbations administered during locomotion on the stance duration. Effect size in the style of Hedges (i.e., taking into account all the variance sources in the model) are shown on the graphs and called $\delta_{t}$. The top-row graph shows a comparison between the standard perturbation protocol ("random") and that including only one mediolateral displacement of the treadmill every $2 \mathrm{~s}$ ("ramp"). (B) The same as in panel(A), but for the swing phase duration. (C) The same as in panel(A), but for the cadence or number of steps per minute. 
bioRxiv preprint doi: https://doi.org/10.1101/2021.04.28.441796; this version posted April 29, 2021. The copyright holder for this preprint (which was not certified by peer review) is the author/funder, who has granted bioRxiv a license to display the preprint in perpetuity. It is made available under aCC-BY 4.0 International license.
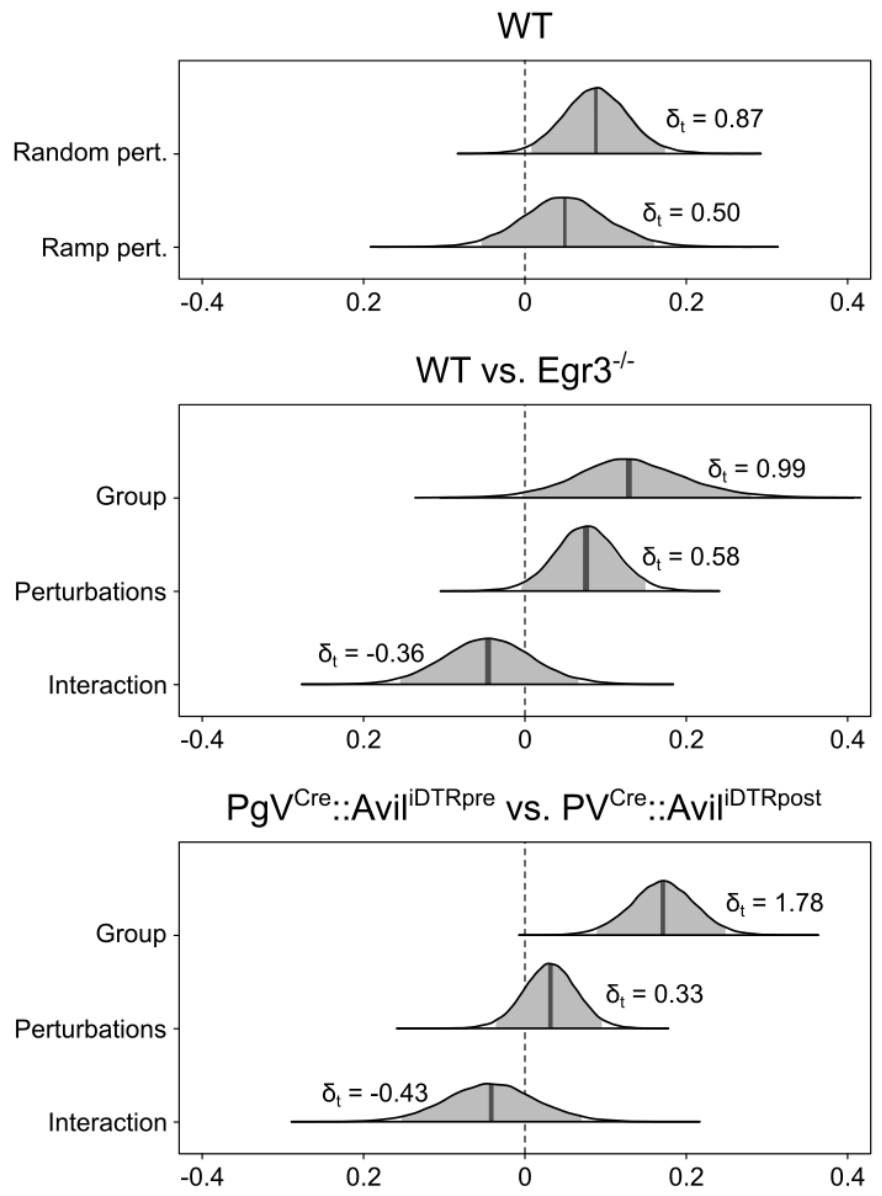

WT vs. WT ${ }^{\mathrm{DCMLL}}$

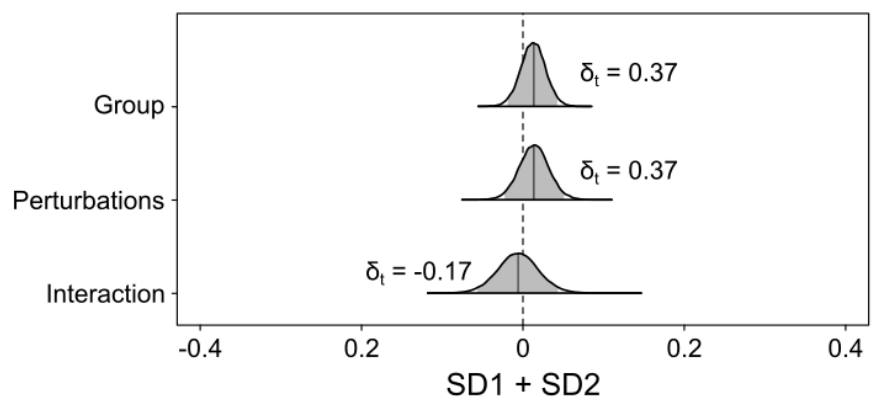

Fig. S7. Posterior $\mathbf{9 5 \%}$ credible intervals for the estimated differences in Poincaré map descriptors. The 95\% credible intervals and their probability distributions (shaded areas) describe the effects and interaction of the investigated groups and perturbations ad ministered during locomotion on the sum of the two Poincaré map descriptors (SD1 and SD2). The top-row graph shows a comparison between the standard perturbation protocol ("random") and that including only one mediolateral displacement of the treadmill every $2 \mathrm{~s}$ ("ramp"). Effect size in the style of Hedges (i.e., taking into account all the variance sources in the model) are shown on the graphs and called $\delta_{t}$. 
bioRxiv preprint doi: https://doi.org/10.1101/2021.04.28.441796; this version posted April 29, 2021. The copyright holder for this preprint (which was not certified by peer review) is the author/funder, who has granted bioRxiv a license to display the preprint in perpetuity. It is made available under aCC-BY 4.0 International license.
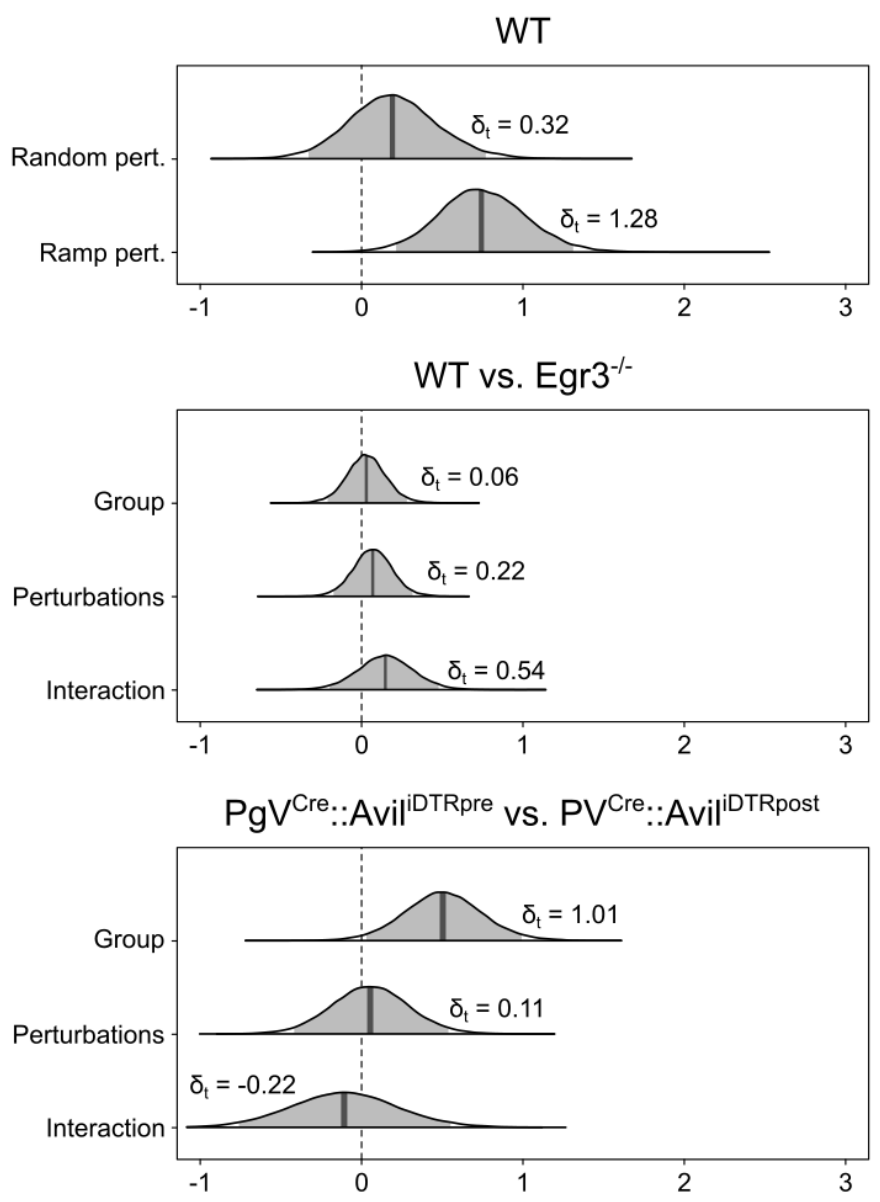

WT vs. WT DCMLL

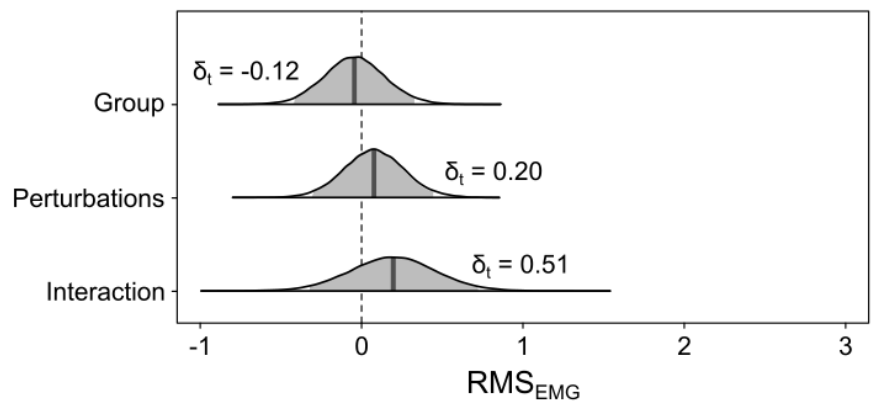

Fig. S8. Posterior $95 \%$ credible intervals for the estimated differences in root mean square of the electromyographic signals $\left(\mathrm{RMS}_{\mathrm{EMG}}\right)$. The $95 \%$ credible intervals and their probability distributions (shaded areas) describe the effects and interaction of the inv estigated groups and perturbations administered during locomotion on the $\mathrm{RMS}_{\mathrm{EMG}}$. The top-row graph shows a comparison between the standard perturbation protocol ("random") and that including only one mediolateral displacement of the treadmill every $2 \mathrm{~s}$ ("ramp"). Effect size in the style of Hedges (i.e., taking into account all the variance sources in the model) are shown on the graphs and called $\delta_{t}$. 
bioRxiv preprint doi: https://doi.org/10.1101/2021.04.28.441796; this version posted April 29, 2021. The copyright holder for this preprint (which was not certified by peer review) is the author/funder, who has granted bioRxiv a license to display the preprint in perpetuity. It is made available under aCC-BY 4.0 International license.

A

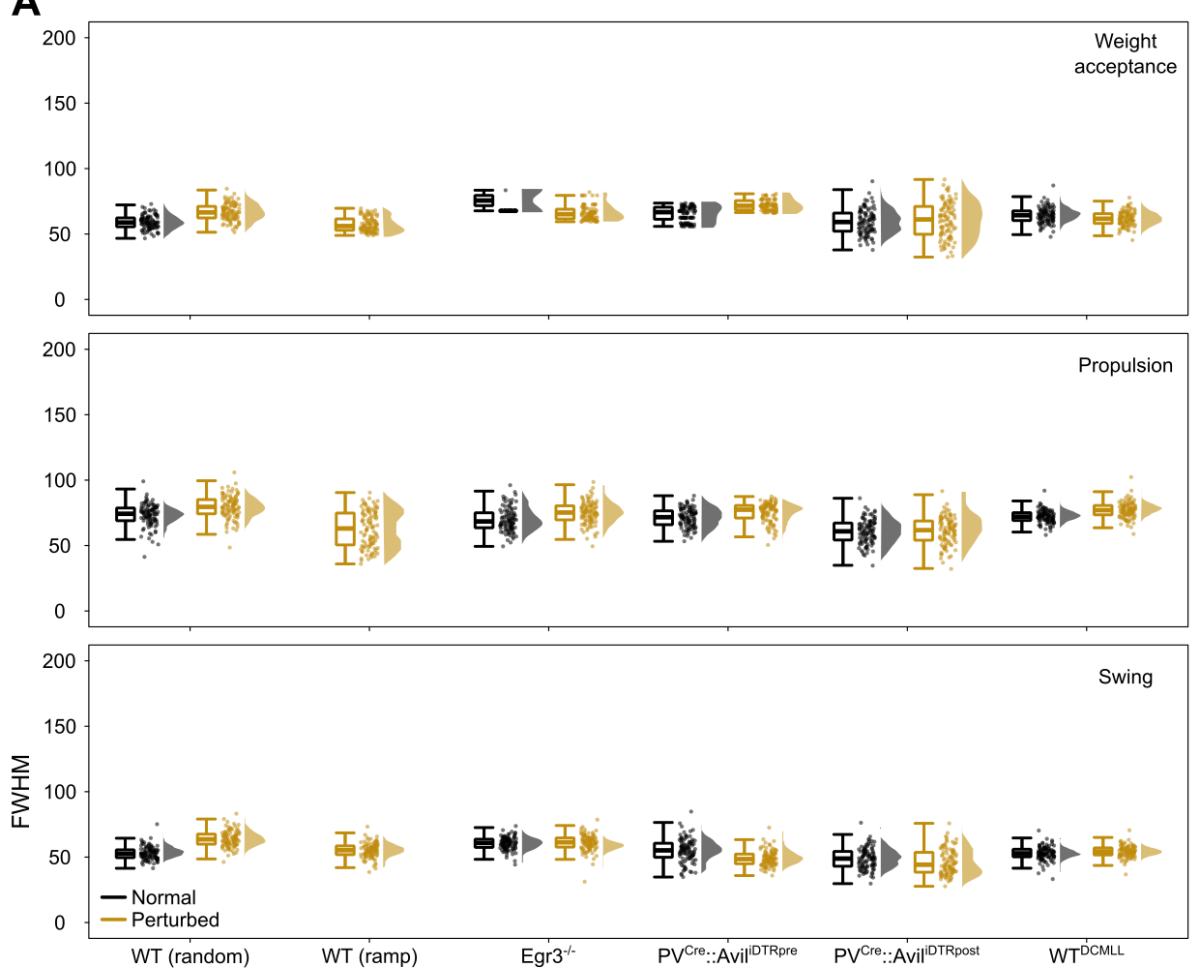

B

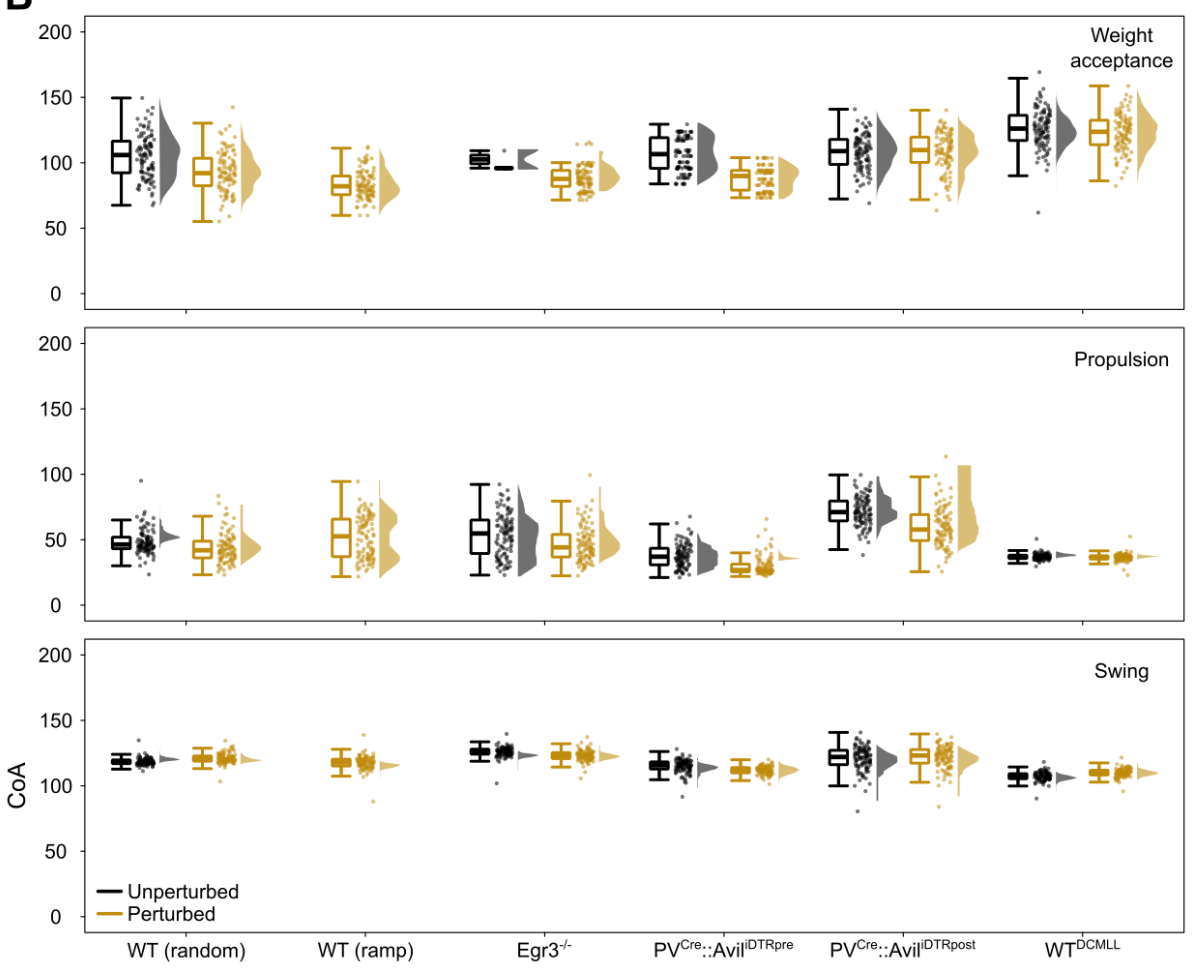

Fig. S9. Full width at half maximum (FWHM) and center of activity (COA) of bootstrapped motor primitives. (A) Boxplots describing the FWHM of the bootstrapped motor primitives (weight acceptance, propulsion and swing) for the investigated groups. Raw data points (each point represents 10 nearest neighbors of the 1000 bootstrapped trials) and their density estimates are presented to the right side of each boxplot. (B) The same as in the previous panel, but for the CoA. 
bioRxiv preprint doi: https://doi.org/10.1101/2021.04.28.441796; this version posted April 29, 2021. The copyright holder for this preprint (which was not certified by peer review) is the author/funder, who has granted bioRxiv a license to display the preprint in perpetuity. It is made available under aCC-BY 4.0 International license.

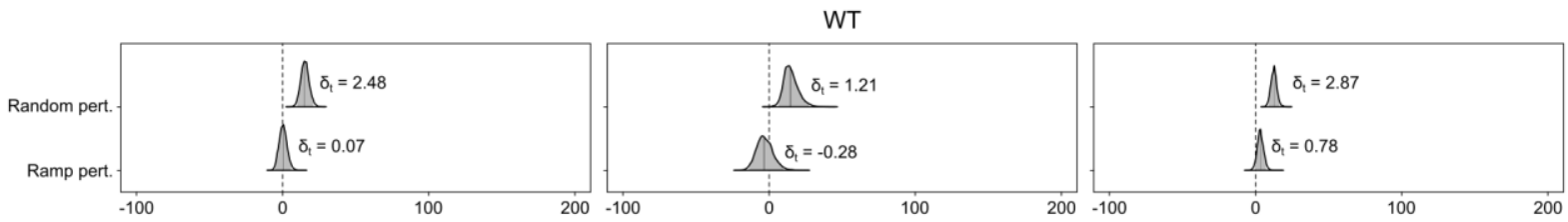

WT vs. Egr3 ${ }^{-1-}$
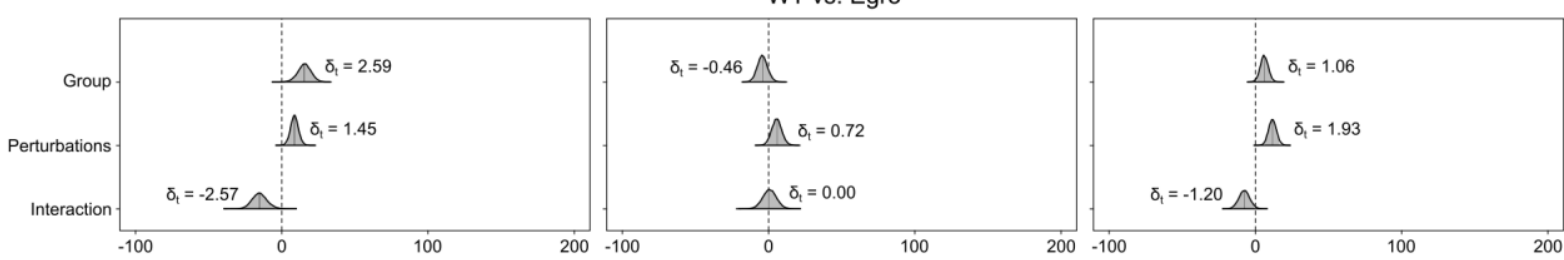

PgVCre::AviliDTRpre vs. PV Cre::AviliDTRpost
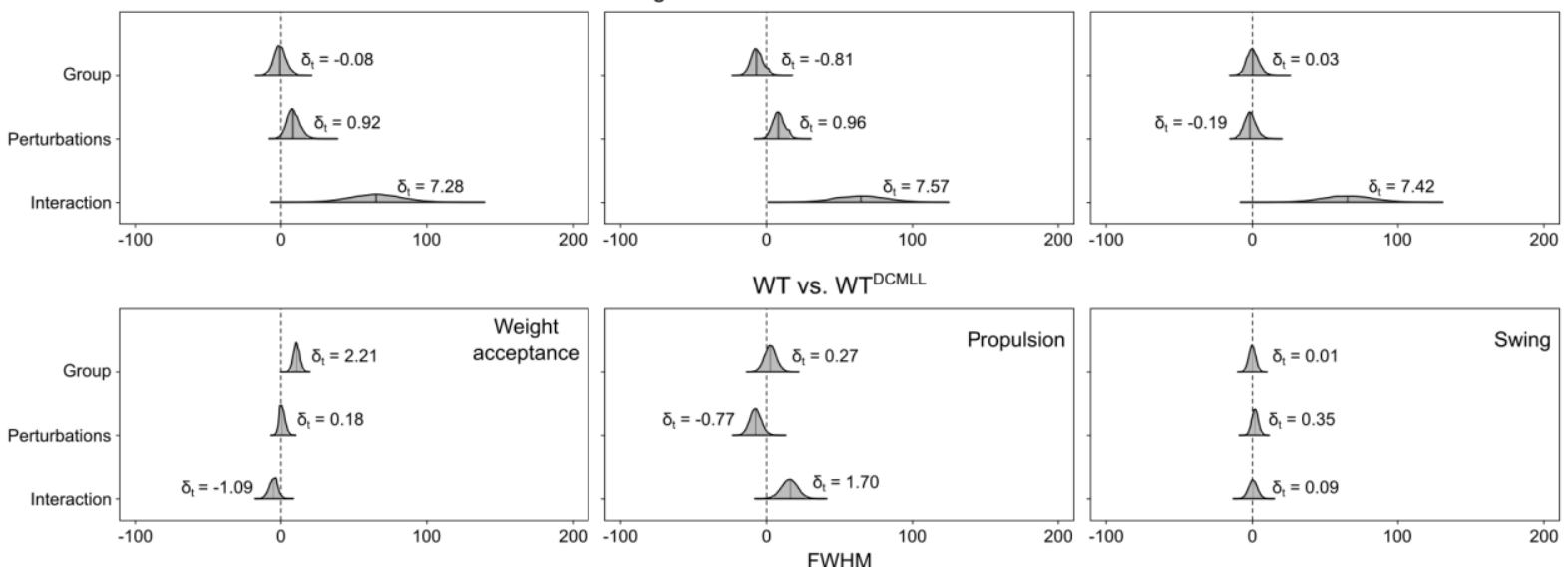

Fig. S10. Posterior $95 \%$ credible intervals for the estimated differences in full width at half maximum (FWHM) of motor primitives. The 95\% credible intervals and their probability distributions (shaded areas) describe the effects and interaction of the investigated groups and perturbations administered during locomotion on the FWHM of motor primitives (left: weight acceptance; middle: propulsion; right: swing).

The top-row graphs show a comparison between the standard perturbation protocol ("random") and that including only one mediolateral displacement of the treadmill every $2 \mathrm{~s}$ ("ramp"). Effect size in the style of Hedges (i.e., taking into account all the variance sources in the model) are shown on the graphs and called $\delta_{\mathrm{t}}$. 
bioRxiv preprint doi: https://doi.org/10.1101/2021.04.28.441796; this version posted April 29, 2021. The copyright holder for this preprint (which was not certified by peer review) is the author/funder, who has granted bioRxiv a license to display the preprint in perpetuity. It is made available under aCC-BY 4.0 International license.
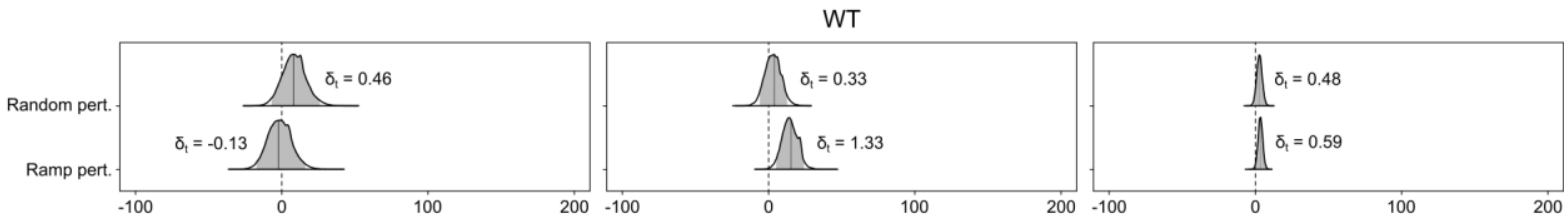

WT vs. Egr3 ${ }^{-1}$
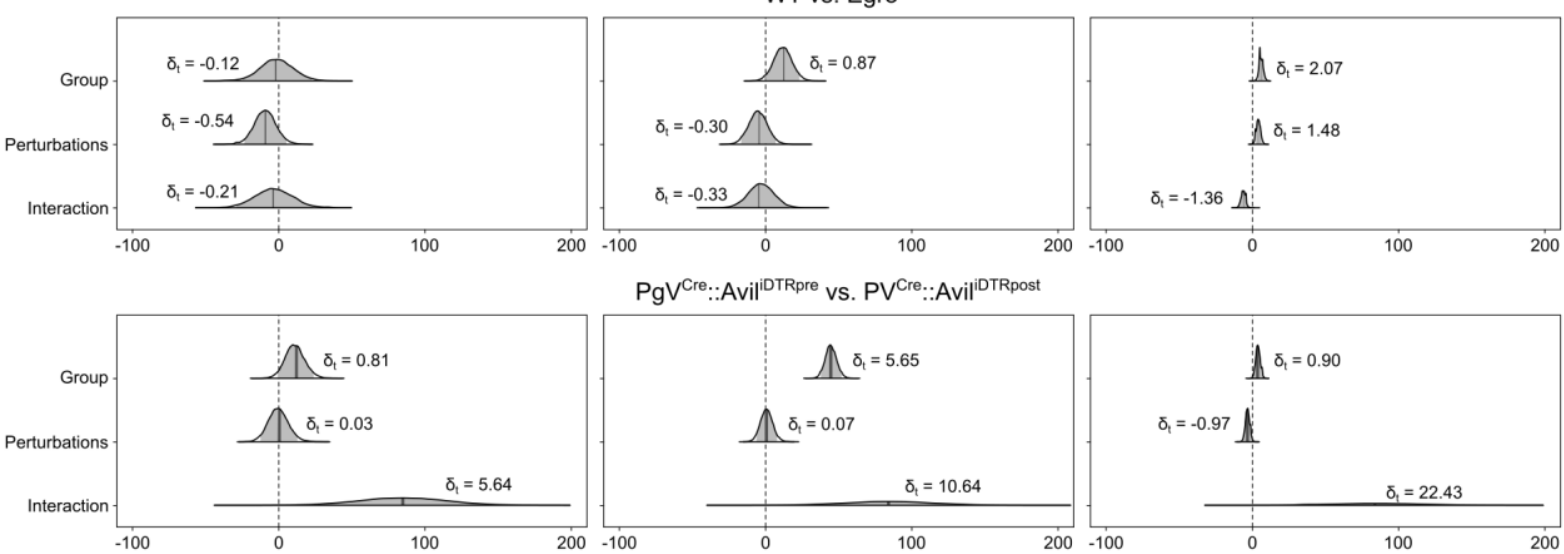

PgV Cre.:Avil ${ }^{\text {DTRpre }}$ vs. PV Cre.::AviliDTRpost
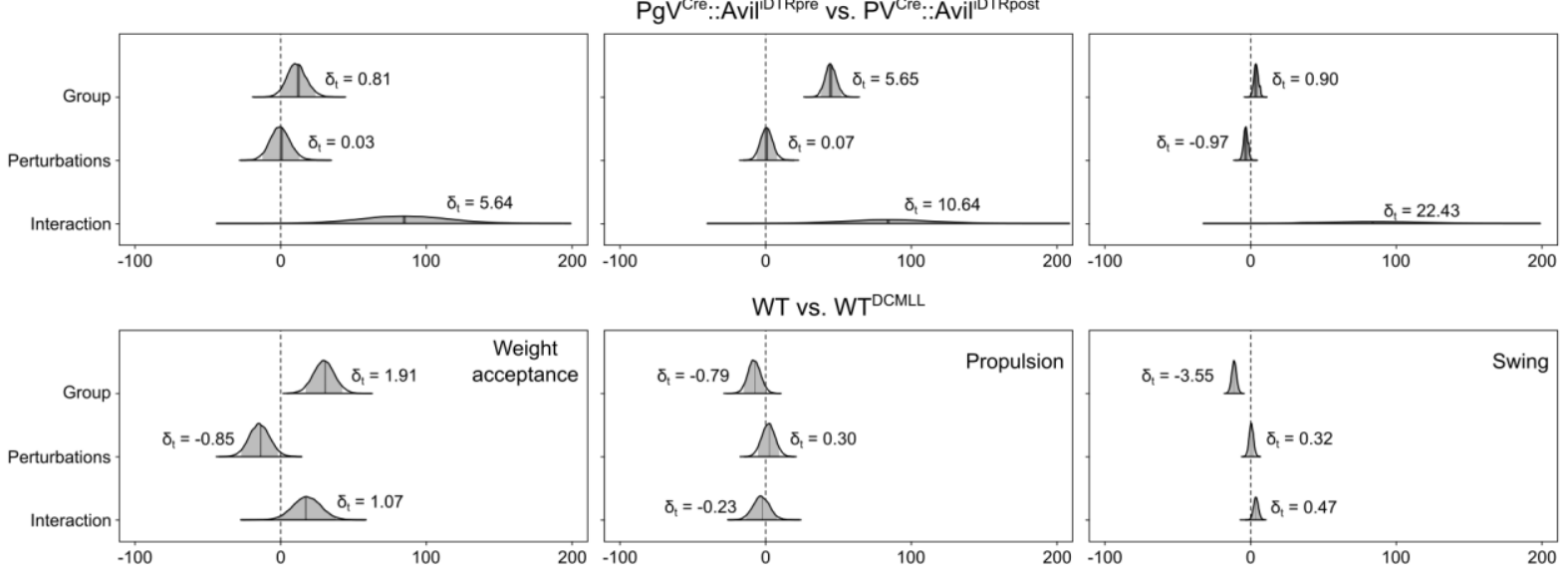

WT vs. WT DCMLL

Fig. S11. Posterior $95 \%$ credible intervals for the estimated differences in center of activity (CoA) of motor primitives. The $95 \%$ credible intervals and their probability distributions (shaded areas) describe the effects and interaction of the investigated groups and perturbations administered during locomotion on the CoA of motor primitives (left: weight acceptance; middle: propulsion; right: swing). The top-row graphs show a comparison between the standard perturbation protocol ("random") and that including only one mediolateral displacement of the treadmill every $2 \mathrm{~s}$ ("ramp"). Effect size in the style of Hedges (i.e., taking into account all the variance sources in the model) are shown on the graphs and called $\delta_{\mathrm{t}}$. 
bioRxiv preprint doi: https://doi.org/10.1101/2021.04.28.441796; this version posted April 29, 2021. The copyright holder for this preprint (which was not certified by peer review) is the author/funder, who has granted bioRxiv a license to display the preprint in perpetuity. It is made available under aCC-BY 4.0 International license.

A

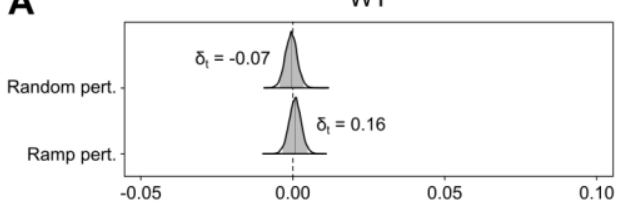

WT vs. Egr3 ${ }^{-1-}$
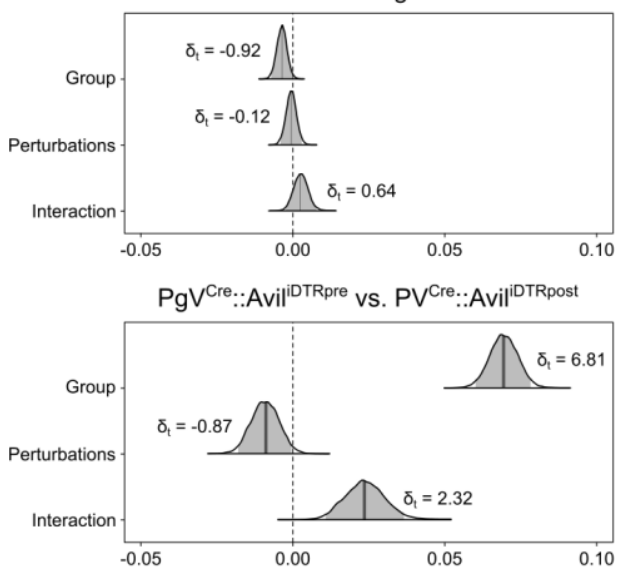

WT vs. WT

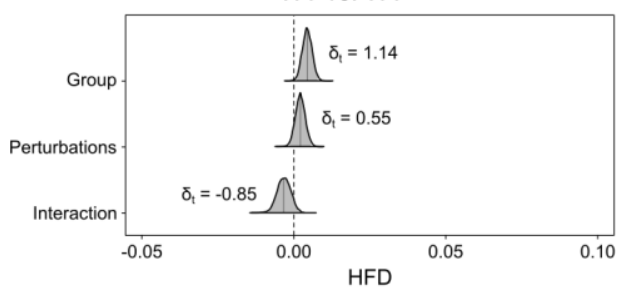

B

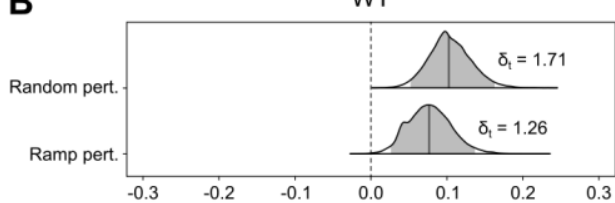

WT vs. Egr3 ${ }^{-/}$

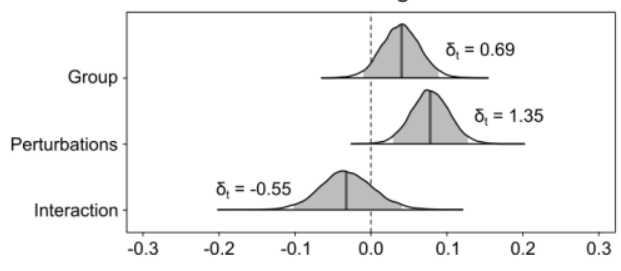

PgV Cre.:.Avili ${ }^{\text {iDRpre }}$ vs. PV Cre.:.Avili ${ }^{\text {DTRpost }}$

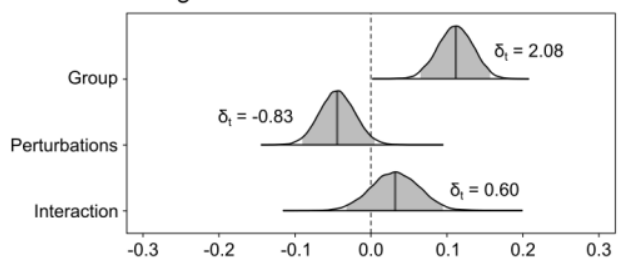

WT vs. WT

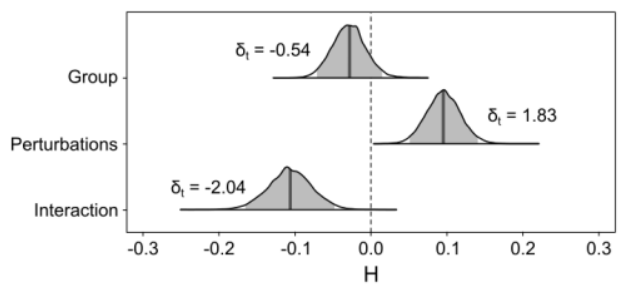

Fig. S12. Posterior $\mathbf{9 5 \%}$ credible intervals for the estimated differences in fractal dimension of motor primitives. (A) The $95 \%$ credible intervals and their probability distributions (shaded areas) describe the effects and interaction of the investigated groups and perturbations administered during locomotion on the Higuchi's fractal dimension (HFD) of motor primitives. The top-row graph shows a comparison between the standard perturbation protocol ("random") and that including only one mediolateral displacement of the treadmill every $2 \mathrm{~s}$ ("ramp"). Effect size in the style of Hedges (i.e., taking into account all the variance sources in the model) are shown on the graphs and called $\delta_{\mathrm{t} \cdot}$ (B) The same as in the previous panel, but for the Hurst exponent $(\mathrm{H})$. 
bioRxiv preprint doi: https://doi.org/10.1101/2021.04.28.441796; this version posted April 29, 2021. The copyright holder for this preprint (which was not certified by peer review) is the author/funder, who has granted bioRxiv a license to display the preprint in perpetuity. It is made available under aCC-BY 4.0 International license.

A
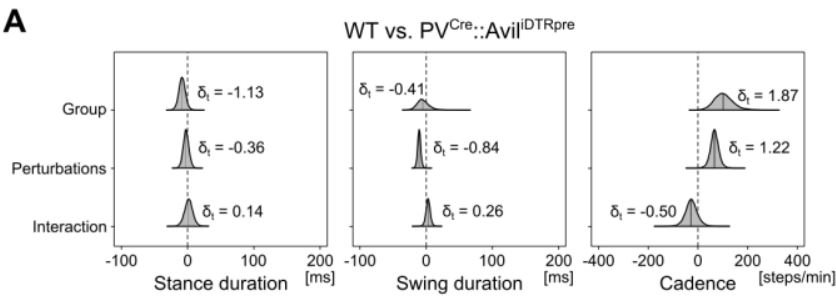

B 二 Unperturbed $=W_{T}$
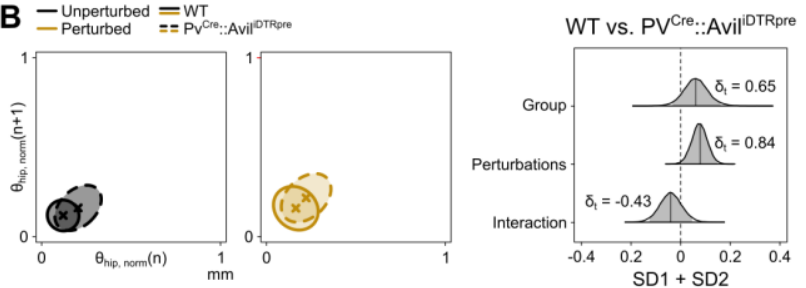

C - Unperturbed $=W T$
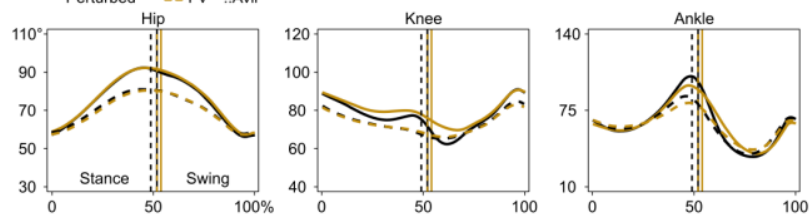

D - Unperturbed $=W T$

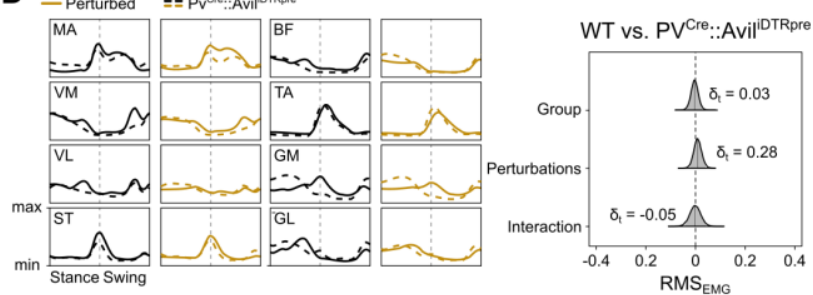

E - Unperturbed $=w T$

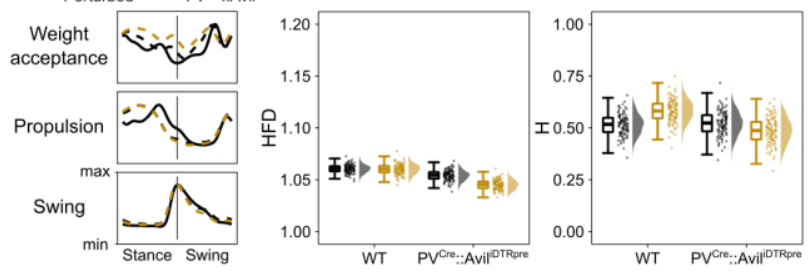

Fig. S13. Largely similar behavior of wild-type and PVCre::Avil ${ }^{\text {DTR }}$ mice, before diphtheria toxin injection. (A) The $95 \%$ credible intervals and their probability distributions (shaded areas) describe the effects and interaction of the investigated groups and perturbations administered during locomotion on the stance duration (left), swing duration (middle) and cadence (right). Effect size in the style of Hedges (i.e., taking into account all the variance sources in the model) are shown on the graphs and called $\delta_{t}$. (B) Poincaré maps (left) of the hip angle $\theta_{\text {hip }}$ at touchdown (n) and $(n+1)$ of all animals, with descriptor ellipse (see methods) and $95 \%$ credible intervals for the Poincaré descrip tors. (C) Average joint angles. (D) Average electromyographic activity of the eight recorded muscles (left) and $95 \%$ credible interv als of the signal's root mean square $\left(\mathrm{RMS}_{\mathrm{EMG}}\right)$. Muscle abbreviations: $\mathrm{MA}=$ gluteus maximus, $\mathrm{VM}=$ vastus medialis, $\mathrm{VL}=$ v. lateralis, $\mathrm{ST}=$ semitendinosus, $\mathrm{BF}=$ biceps femoris, $\mathrm{TA}=$ tibialis anterior, $\mathrm{GM}=$ gastrocnemius medialis, and $\mathrm{GL}=$ g. lateralis. (E) Average motor primitives (left) and their complexity (right). Boxplots describe the Higuchi's fractal dimension (HFD) and Hurst exponent $(\mathrm{H})$ of the bootstrapped primitives. Raw data points (each point represents 10 nearest neighbors of the 1000 bootstrapped trials) and their density estimates are presented to the right side of each boxplot. 
bioRxiv preprint doi: https://doi.org/10.1101/2021.04.28.441796; this version posted April 29, 2021. The copyright holder for this preprint (which was not certified by peer review) is the author/funder, who has granted bioRxiv a license to display the preprint in perpetuity. It is made available under aCC-BY 4.0 International license.

A
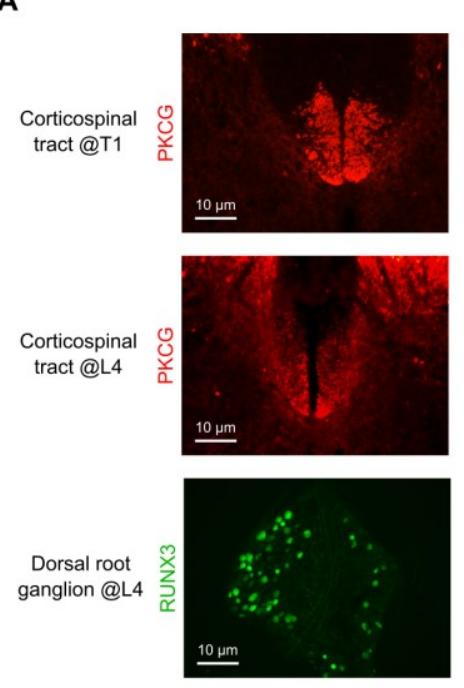

C

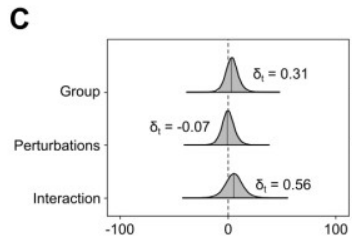

\section{B}

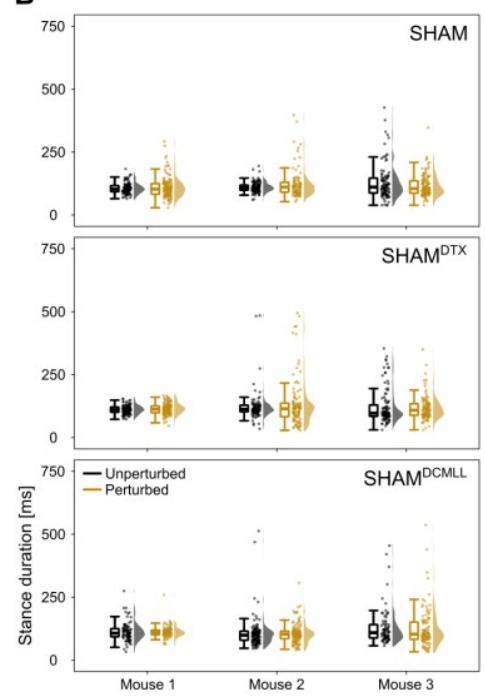

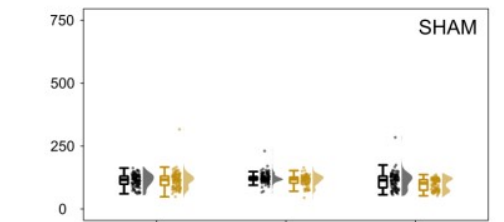
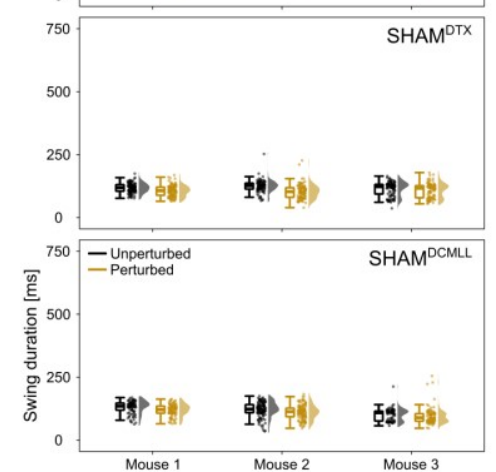

SHAM vs. SHAM ${ }^{\text {DTX }}$

D SHAM vs. SHAM ${ }^{\text {DTX }}$

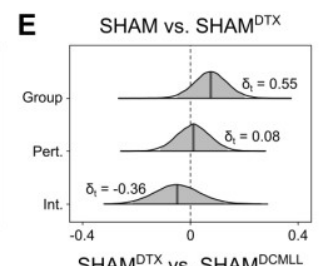

SHAM $^{\text {DTX }}$ vs. SHAM ${ }^{\text {DCMLL }}$
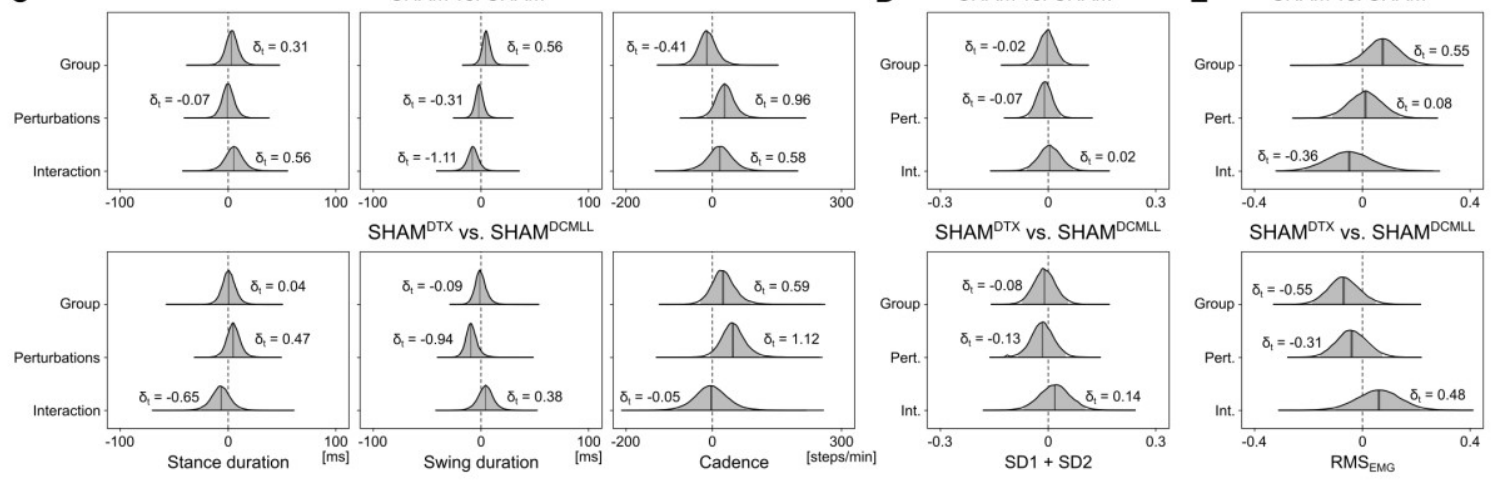

Fig. S14. Sham experiments for diphtheria toxin (DTX) injections and dorsal column-medial lemniscus pathway lesion (DCMLL). (A) For the sham experiments, three animals underwent both DTX injection and DCMLL surgery (lesion excluded). Protein kinase C gamma (PKCG) is expressed in the axons of the corticospinal tract (CST). Here, both rostral (spinal segment T1) and caudal (L4) sections with respect to the sham surgery site show intact CST. Runt-related transcription factor 3 (RUNX3) is selectively expressed in intact proprioceptive sensory neurons located in dorsal root ganglia. After DTX injection in sham mice that do not express human DTX receptors, the proprioceptive neuron terminals are left intact. (B). Stance (left) and swing (right) phase duration for each step recorded in every animal used for the sham experiments. Individual step values, pictured as dots, and their distributions are shown next to the relevant boxplot. (C). The $95 \%$ credible intervals and their probability distributions (shaded areas) describe the effects and interaction of the inv estigated conditions and perturbations administered during locomotion on the stance duration (left), swing duration (middle) and cadence (right). Effect size in the style of Hedges (i.e., taking into account all the variance sources in the model) are shown on the graphs and called $\delta_{\text {t. }}(\mathbf{D})$. The same as in $(\mathbf{C})$, but for the two Poincaré map descriptors (SD1 and SD2). (E) The same as in (C), but for the root mean square of the electromyographic signals $\left(\mathrm{RMS}_{\mathrm{EMG}}\right)$. 


\section{References and Notes:}

1. T. G. Brown, The intrinsic factors in the act of progression in the mammal. Proc. R. Soc. London. Ser. B, Contain. Pap. a Biol. Character. 84, 308-319 (1911).

2. S. K. Karadimas, K. Satkunendrarajah, A. M. Laliberte, D. Ringuette, I. Weisspapir, L. Li, S. Gosgnach, M. G. Fehlings, Sensory cortical control of movement. Nat. Neurosci. 23 (2019), doi:10.1038/s41593-019-0536-7.

3. S. Rossignol, R. Dubuc, J.-P. Gossard, Dynamic Sensorimotor Interactions in Locomotion. Physiol. Rev. 86, 89-154 (2006).

4. F. Cignetti, A. Fontan, J. Menant, B. Nazarian, J. L. Anton, M. Vaugoyeau, C. Assaiante, Protracted Development of the Proprioceptive Brain Network During and Beyond Adolescence. Cereb. cortex. 27, 1285-1296 (2017).

5. T. Akay, Sensory Feedback Control of Locomotor Pattern Generation in Cats and Mice. Neuroscience. 450, 161-167 (2020).

6. S. Grillner, A. El Manira, Current Principles of Motor Control, with Special Reference to Vertebrate Locomotion. Physiol. Rev. 100, 271-320 (2020).

7. A. Santuz, T. Akay, W. P. Mayer, T. L. Wells, A. Schroll, A. Arampatzis, Modular organization of murine locomotor pattern in the presence and absence of sensory feedback from muscle spindles. J. Physiol. 597, 3147-3165 (2019).

8. J. C. Tuthill, E. Azim, Proprioception. Curr. Biol. 28, R194-R203 (2018).

9. K. G. Pearson, Proprioceptive regulation of locomotion. Curr. Opin. Neurobiol. 5, 786791 (1995).

10. T. Akay, W. G. Tourtellotte, S. Arber, T. M. Jessell, Degradation of mouse locomotor pattern in the absence of proprioceptive sensory feedback. Proc. Natl. Acad. Sci. U. S. A. 111, 16877-82 (2014).

11. A. Takeoka, S. Arber, Functional local proprioceptive feedback circuits initiate and maintain locomotor recovery after spinal cord injury. Cell Rep. 27, 71-85.e3 (2019).

12. W. P. Mayer, A. J. Murray, S. Brenner-Morton, T. M. Jessell, W. G. Tourtellotte, T. Akay, Role of muscle spindle feedback in regulating muscle activity strength during walking at different speed in mice. J. Neurophysiol. 120, 2484-2497 (2018).

13. A. W. Hantman, T. M. Jessell, Clarke's column neurons as the focus of a corticospinal corollary circuit. Nat. Neurosci. 13, 1233-1239 (2010).

14. E. Bizzi, V. C.-K. Cheung, A. D’Avella, P. Saltiel, M. C. Tresch, Combining modules for movement. Brain Res. Rev. 57, 125-33 (2008).

15. D. D. Lee, H. S. Seung, Learning the parts of objects by non-negative matrix factorization. Nature. 401, 788-91 (1999).

16. A. Santuz, A. Ekizos, Y. Kunimasa, K. Kijima, M. Ishikawa, A. Arampatzis, Lower complexity of motor primitives ensures robust control of high-speed human locomotion. Heliyon. 6, e05377 (2020).

17. J. Niu, L. Ding, J. J. Li, H. Kim, J. Liu, H. Li, A. Moberly, T. C. Badea, I. D. Duncan, Y. J. Son, S. S. Scherer, W. Luo, Modality-based organization of ascending somatosensory 
axons in the direct dorsal column pathway. J. Neurosci. 33, 17691-17709 (2013).

18. W. G. Tourtellotte, J. Milbrandt, Sensory ataxia and muscle spindle agenesis in mice lacking the transcription factor Egr3. Nat. Genet. 20, 87-91 (1998).

19. S. Hippenmeyer, E. Vrieseling, M. Sigrist, T. Portmann, C. Laengle, D. R. Ladle, S. Arber, A developmental switch in the response of DRG neurons to ETS transcription factor signaling. PLoS Biol. 3, 0878-0890 (2005).

20. K. K. Stantcheva, L. Iovino, R. Dhandapani, C. Martinez, L. Castaldi, L. Nocchi, E. Perlas, C. Portulano, M. Pesaresi, K. S. Shirlekar, F. Castro Reis, T. Paparountas, D. Bilbao, P. A. Heppenstall, A subpopulation of itch-sensing neurons marked by Ret and somatostatin expression. EMBO Rep. 17, 585-600 (2016).

21. V. H. Sousa, G. Miyoshi, J. Hjerling-Leffler, T. Karayannis, G. Fishell, Characterization of Nkx6-2-derived neocortical interneuron lineages. Cereb. Cortex. 19, 1-10 (2009).

22. A. Santuz, T. Akay, Fractal analysis of muscle activity patterns during locomotion: pitfalls and how to avoid them. J. Neurophysiol. 124, 1083-1091 (2020).

23. K. G. Pearson, H. J. Acharya, K. Fouad, A new electrode configuration for recording electromyographic activity in behaving mice. J. Neurosci. Methods. 148, 36-42 (2005).

24. T. Akay, H. J. Acharya, K. Fouad, K. G. Pearson, Behavioral and Electromyographic Characterization of Mice Lacking EphA4 Receptors. J. Neurophysiol. 96, 642-651 (2006).

25. M. Harrison, A. O’Brien, L. Adams, G. Cowin, M. J. Ruitenberg, G. Sengul, C. Watson, Vertebral landmarks for the identification of spinal cord segments in the mouse. Neuroimage. 68, 22-29 (2013).

26. A. Mathis, P. Mamidanna, K. M. Cury, T. Abe, V. N. Murthy, M. W. Mathis, M. Bethge, DeepLabCut: markerless pose estimation of user-defined body parts with deep learning. Nat. Neurosci. 21, 1281-1289 (2018).

27. K. He, X. Zhang, S. Ren, J. Sun, Deep residual learning for image recognition. Proc. IEEE Comput. Soc. Conf. Comput. Vis. Pattern Recognit. 2016-Decem, 770-778 (2016).

28. E. Insafutdinov, L. Pishchulin, B. Andres, M. Andriluka, B. Schiele, in European Conference on Computer Vision, B. Leibe, J. Matas, N. Sebe, M. Welling, Eds. (Springer International Publishing, Cham, 2016; http://link.springer.com/10.1007/978-3-319-464664), vol. 9910 of Lecture Notes in Computer Science, pp. 34-50.

29. A. Santuz, A. Ekizos, N. Eckardt, A. Kibele, A. Arampatzis, Challenging human locomotion: stability and modular organisation in unsteady conditions. Sci. Rep. 8, 2740 (2018).

30. C. Maiwald, T. Sterzing, T. A. Mayer, T. L. Milani, Detecting foot-to-ground contact from kinematic data in running. Footwear Sci. 1, 111-118 (2009).

31. M. Brennan, M. Palaniswami, P. Kamen, Do existing measures of Poincare plot geometry reflect nonlinear features of heart rate variability? IEEE Trans. Biomed. Eng. 48, 13421347 (2001).

32. A. Santuz, A. Ekizos, L. Janshen, V. Baltzopoulos, A. Arampatzis, On the Methodological Implications of Extracting Muscle Synergies from Human Locomotion. Int. J. Neural 
Syst. 27, 1750007 (2017).

33. A. Santuz, A. Ekizos, L. Janshen, F. Mersmann, S. Bohm, V. Baltzopoulos, A. Arampatzis, Modular Control of Human Movement During Running: An Open Access Data Set. Front. Physiol. 9, 1509 (2018).

34. A. Santuz, O. Laflamme, T. Akay, Differential role of spinal and supraspinal processing of proprioceptive information in mouse locomotion. Zenodo Dataset (2021), doi: $10.5281 /$ zenodo.4724766.

35. T. Higuchi, Approach to an irregular time series on the basis of the fractal theory. Phys. $D$ Nonlinear Phenom. 31, 277-283 (1988).

36. H. E. Hurst, Long-term storage capacity of reservoirs. Trans. Am. Soc. Civ. Eng. 116, 770-808 (1951).

37. B. B. Mandelbrot, J. R. Wallis, Robustness of the rescaled range R/S in the measurement of noncyclic long run statistical dependence. Water Resour. Res. 5, 967-988 (1969).

38. C. J. Anmuth, G. Goldberg, N. H. Mayer, Fractal dimension of electromyographic signals recorded with surface electrodes during isometric contractions is linearly correlated with muscle activation. Muscle Nerve. 17, 953-954 (1994).

39. S. Kesić, S. Z. Spasić, Application of Higuchi's fractal dimension from basic to clinical neurophysiology: A review. Comput. Methods Programs Biomed. 133, 55-70 (2016).

40. T. Gneiting, M. Schlather, Stochastic Models That Separate Fractal Dimension and the Hurst Effect. SIAM Rev. 46, 269-282 (2004).

41. B. B. Mandelbrot, The Fractal Geometry of Nature (W. H. Freeman and Co., New York, 1983).

42. B. Qian, K. Rasheed, in Proceedings of the Second IASTED International Conference on Financial Engineering and Applications (2004), pp. 203-209.

43. P. C. Bürkner, brms: An R package for Bayesian multilevel models using Stan. J. Stat. Softw. 80 (2017), doi:10.18637/jss.v080.i01.

44. B. Carpenter, A. Gelman, M. D. Hoffman, D. Lee, B. Goodrich, M. Betancourt, M. A. Brubaker, J. Guo, P. Li, A. Riddell, Stan: A probabilistic programming language. J. Stat. Softw. 76 (2017), doi:10.18637/jss.v076.i01.

45. L. Nalborczyk, C. Batailler, H. Loevenbruck, A. Vilain, P. C. Bürkner, An introduction to bayesian multilevel models using brms: A case study of gender effects on vowel variability in standard Indonesian. J. Speech, Lang. Hear. Res. 62, 1225-1242 (2019).

46. T. C. Pataky, One-dimensional statistical parametric mapping in Python. Comput. Methods Biomech. Biomed. Engin. 15, 295-301 (2012).

Acknowledgments: The authors are grateful to Brenda Ross for helping with the surgeries and maintaining the mouse colony, to Rachel Banks and Tyler Wells for supporting electrode production and tissue preparation, to Katrina Meyer for the immunohistochemistry suggestions and to Jacques Duysens and Sten Grillner for their comments on the manuscript. We also thank the Jessell lab, particularly Susan Brenner-Morton for sharing their primary antibodies against RUNX3 and VGLUT1 and Warren Tourtellotte for providing the Egr $3^{-/}$breeder mice. Funding: 
This research was funded by the Canadian Institutes of Health Research (Project Grant PJT162357); A. Santuz was funded through the Research Fellowship Program (SA 3695/1-1) awarded by the Deutsche Forschungsgemeinschaft (DFG); Author contributions:

Conceptualization: A.S. and T.A.; Data curation: A.S.; Formal analysis: A.S.; Investigation: A.S. and O.L.; Software: A.S.; Supervision: T.A.; Visualization: A.S.; Writing - original draft: A.S.; Writing - review \& editing: A.S., O.L. and T.A.; Competing interests: Authors declare no competing interests; Data and materials availability: All datasets generated and analyzed during the current study and an exhaustive explanation of their structure are available at the Zenodo repository (DOI: 10.5281/zenodo.4724766). 